\title{
The Novel P330L Pathogenic Variant of Aromatic Amino Acid Decarboxylase Maps on the Catalytic Flexible Loop Underlying its Crucial Role
}

\section{Giovanni Bisello}

University of Verona: Universita degli Studi di Verona

\section{Katarzyna Kusmierska}

Medical University of Warsaw: Warszawski Uniwersytet Medyczny

Marcel M. Verbeek

Radboud Universiteit

Jolanta Sykut-Cegielska

Medical University of Warsaw: Warszawski Uniwersytet Medyczny

Michèl A.A.P. Willemsen

Radboud Universiteit

\section{Ron A. Wevers}

Radboud Universiteit

\section{Krystyna Szymańska}

Medical University of Warsaw: Warszawski Uniwersytet Medyczny

Jarosław Poznanski

Polish Academy of Sciences: Polska Akademia Nauk

Jakub Drozak

University of Warsaw: Uniwersytet Warszawski

Katarzyna Wertheim-Tysarowska

University of Warsaw: Uniwersytet Warszawski

\section{Agnieszka Magdalena Rygiel}

University of Warsaw: Uniwersytet Warszawski

Mariarita Bertoldi ( $\square$ mita.bertoldi@univr.it)

University of Verona: Universita degli Studi di Verona https://orcid.org/0000-0002-2337-9928

\section{Research Article}

Keywords: Pyridoxal 5'-phosphate, aromatic amino acid decarboxylase, aromatic amino acid decarboxylase deficiency, monoamine neurotransmitter disorder, catalytic loop, structure and function studies 
Posted Date: March 7th, 2022

DOI: https://doi.org/10.21203/rs.3.rs-1395907/v1

License: (c) (i) This work is licensed under a Creative Commons Attribution 4.0 International License. Read Full License 


\section{The novel P330L pathogenic variant of aromatic amino acid decarboxylase maps on the catalytic flexible loop underlying its crucial role}

Giovanni Bisello ${ }^{1}$, Katarzyna Kusmierska², Marcel M. Verbeek ${ }^{3,4}$, Jolanta Sykut Cegielska $^{5}$, Michèl A.A.P. Willemsen ${ }^{6}$, Ron A. Wevers ${ }^{4}$, Krystyna Szymańska ${ }^{7}$, Jarosław Poznanski ${ }^{8}$, Jakub Drozak ${ }^{9}$, Katarzyna Wertheim-Tysarowska ${ }^{10}$, Agnieszka Magdalena Rygie ${ }^{10}$ and Mariarita Bertoldi ${ }^{*}$

${ }^{1}$ Department of Neuroscience, Biomedicine and Movement Sciences, Section of Biochemistry, Strada Le Grazie 8, 37134 Verona, Italy

${ }^{2}$ Department of Screening and Metabolic Diagnostics, Institute of Mother and Child, Warsaw, Poland ${ }^{3}$ Department of Neurology, Donders Institute for Brain, Cognition and Behaviour, Radboud University Medical Centre, Geert Grooteplein 10, 6525 GA Nijmegen, The Netherlands

${ }^{4}$ Translational Metabolic Laboratory, Department Laboratory Medicine, Radboud University Medical Cente, Geert Grooteplein 10, 6525 GA Nijmegen, The Netherlands

${ }^{5}$ Department of Inborn Errors of Metabolism and Paediatrics, Institute of Mother and Child, Warsaw, Poland

${ }^{6}$ Department of Pediatric Neurology, Radboud University Medical Centre, Geert Grooteplein 10, 6525 GA Nijmegen, The Netherlands

${ }^{7}$ Department of Child and Adolescent Psychiatry, Medical University of Warsaw, Warsaw, Poland

${ }^{8}$ Department of Biophysics, Institute of Biochemistry and Biophysics, Polish Academy of Sciences, Warsaw, Poland

${ }^{9}$ Department of Metabolic Regulation, Faculty of Biology, University of Warsaw, Warsaw, Poland ${ }^{10}$ Department of Medical Genetics, Institute of Mother and Child, Warsaw, Poland

*Correspondence: mita.bertoldi@univr.it (MB)

ORCID ID:

Mariarita Bertoldi: 0000-0002-2337-9928 


\begin{abstract}
Aromatic amino acid decarboxylase (AADC) deficiency is a rare monogenic disease, often fatal in the first decade, causing severe intellectual disability, movement disorders and autonomic disfunction. It is due to mutations in the gene coding for the AADC enzyme responsible for the synthesis of the important neurotransmitters dopamine, norepinephrine, epinephrine and serotonin. Using whole exome sequencing, we have identified a novel homozygous c989C $>$ T (Pro330Leu) variant of AADC causing AADC deficiency. Pro330 is part of an essential structural and functional element: the flexible catalytic loop suggested to cover the active site crevice as a lid and properly position the catalytic residues. Our investigations provide evidence that Pro330 concurs in the achievement of an optimal catalytic competence. Through a combination of bioinformatic approaches, dynamic light scattering measurements, limited proteolysis experiments, spectroscopic and in solution analyses, we demonstrate that the substitution of Pro330 with Leu, although not determining gross conformational changes, results in an enzymatic species that is highly affected in catalysis. This defect does not lead to active site structural disassembling, nor to the inability to bind the pyridoxal 5'-phosphate (PLP) cofactor. The molecular basis for the pathogenic effect of this variant is rather due to a mispositioning of the catalytically competent external aldimine intermediate, as corroborated by spectroscopic analyses and $\mathrm{pH}$ dependence of the kinetic parameters. Altogether, we determined the structural basis for the severity of the manifestation of AADC deficiency in this patient and discussed the rationale for a precision therapy.
\end{abstract}

\title{
Keywords
}

Pyridoxal 5'-phosphate, aromatic amino acid decarboxylase, aromatic amino acid decarboxylase deficiency, monoamine neurotransmitter disorder, catalytic loop, structure and function studies

\footnotetext{
Abbreviations

PLP, pyridoxal 5'-phosphate; PMP, pyridoxamine 5'-phosphate; CL, catalytic loop; AADC, aromatic amino acid decarboxylase; L-Dopa, L-3,4-dihydroxyphenylalanine; L-5HTP, L-5hydroxytryptophan; DME, L-3,4-dihydroxyphenylalanine methyl ester; cDopa, carbiDopa; GAD, glutamate decarboxylase; HDC, histidine decarboxylase; CSAD, cysteine sulfinic acid decarboxylase; CSF, cerebrospinal fluid; HVA, homovanillic acid; 5-HIAA, 5-hydroxyindoleacetic acid; 3-OMD, 3-orthomethyl dopa; 5-HTF, 5-methyltetrahydrofolate; VLA, vanillactic acid; AVA, acetyl vanilalanine
} 


\section{Introduction}

A common hallmark among pyridoxal 5'-phosphate (PLP) $\alpha$-decarboxylases of Group II [1] is the presence of a catalytic loop (CL) essential for enzymatic activity [2]. These enzymes play important roles in all tissues and many of them are responsible for the synthesis of important monoamine neurotransmitters. Structurally, they all belong to the aspartate aminotransferase family (or Fold-Type I) $[3,4]$ of PLP-dependent enzymes and are functional asymmetric homodimers with the CL of each subunit exposed to the solvent and able to insert into the opposite active site in the so-called active closed enzymatic form (Supplemental Results). One of the best characterized among them is mammalian aromatic amino acid decarboxylase (AADC) responsible for the synthesis of dopamine and serotonin from L-3,4-dihydroxyphenylalanine (L-Dopa) and L-5-hydroxytryptophan (L-5HTP), respectively [5]. The spatial structure of pig holoAADC has been solved in the absence and in the presence of the inhibitor carbiDopa (cDopa) [6] that, although irreversibly bound to PLP through its hydrazine group, mimics the substrate binding mode, especially in the positioning of the catechol moiety. The structure of the human apoAADC has also been solved [7] in an unusual open assembly that could account for the mechanism of PLP binding and apoprotein preferential degradation [7]. The global holoAADC architecture is organized in two interlocked monomers, each composed by a N-terminal domain (NTD, residues 1-85), a large domain containing the PLP cofactor (residues 86360) and a C-terminal domain (CTD, residues 361-480). The CL (residues 328-339) is part of the loop3 (residues 323-357) belonging to the large domain and is assumed to protrude inside the opposite active site during catalysis in the dimeric functional unit [2]. This flexible stretch is visible only in few spatial structures of $\alpha$-decarboxylases solved so far $[2,8]$ but not in mammalian AADC. The highly solvent exposed nature of the $\mathrm{CL}$ in $\alpha$-decarboxylases is corroborated by the limited proteolysis susceptibility displayed by AADC [9], human glutamate decarboxylase (GAD) isoforms [10] and human histidine decarboxylase (HDC) [11]. Importantly, the CL contains the catalytic tyrosine residue [12] implicated in the enzymatic mechanism of decarboxylation [8, 11-13] and proposed to be properly oriented towards the $\mathrm{C} \alpha$ of the substrate (as it was later demonstrated for HDC [11] and GAD67 [13] and seen in human cysteine sulfinic acid decarboxylase (CSAD) [2], as well as in plant AADCs [8]). Thus, the flexibility of the CL appears crucial for the orientation of key residues for activity. Among the evolutionary related $\alpha$-decarboxylases with solved CL, human HDC shares the highest sequence identity (52.1\%) with AADC and has been employed to build a model of the whole pig AADC [14]. Here it is evident how residues belonging to loop3 such as Asp345 or Arg347 concur in a network of interactions with the CL, to properly position it inside the facing active site [14]. 
The latest release of AlphaFold2 [15, 16] could provide us with a model of the human enzyme. However, some reasons prevented us to use it. First, AlphaFold2 models do not show coenzymes or bound ligands and this is detrimental, especially for multimeric enzymes having shared active sites. Secondly, despite the undoubtful advantage to visualize so far invisible parts of a protein, disordered regions are displayed at a low confidence level, especially when they find at the interface in quaternary structures. For this reason, as recommended by structural biologists [17], caution should be paid in interpreting AlphaFold2 modeled mobile regions.

Here we present the first pathogenic variant of $D D C$ mapping in the CL of AADC. Mutations (substitutions, deletions, insertions, splicing) in the DDC gene coding for AADC (GRCh38.p13; chr7:50,447,733-50,576,163) lead to AADC deficiency (OMIM\#608643), a monogenic neurometabolic disease, determining a complex severe phenotype [18-20] which is often fatal in the first decade. Up to now, over 90 mutations are known and more than 130 patients identified in both homozygosis and heterozygosis [20]. In the last few years, we have characterized more than 30 variants carrying amino acid substitutions [14, 21-26]. Mutations are spread over the entire gene sequence but none has been identified on the CL until now. Notably, some pathogenic amino acid substitutions map on loop3 [2, 7] and have been already characterized: R347Q [21], R347G [14] and L353P [23]. These variants share an almost invariant structural fold as the wild-type (WT) enzyme but a remarkable loss of catalytic efficiency attributed to the proximity of the replacements to the active site possibly altering CL engagement into catalysis.

Here, we report the case of a homozygous patient carrying for the first time a substitution, the novel P330L, mapping on the CL of AADC and characterize the structural and functional properties of the P330L AADC protein. Altogether, we propose that the molecular basis for the lack of catalytic activity of the P330L variant consists in the impairment of CL closing to reach a catalytically competent conformation. More precisely, the P330L protein pertains all the structural requirements of the WT counterpart in terms of secondary and tertiary structure as well as in coenzyme binding affinity, but reveals defects in productively orienting the external aldimine catalytic intermediate. This translates into a species unable to produce neurotransmitters at sufficient levels. Patients carrying mutations affecting this element of the protein could be responsive to dopamine agonists and MAOinhibitors with less pronounced benefits from vitamin B6 and L-Dopa treatments and could be possibly considered as candidates for gene therapy having an almost inactive AADC enzyme. 


\section{Materials and Methods}

\section{Materials}

PLP, L-Dopa, dopamine, cDopa, dopa methylester (DME), L-5-hydroxytryptophan (L-5HTP), serotonin, hydroxylamine hydrochloride, isopropyl- $\beta-\mathrm{D}$ thiogalactopyranoside (IPTG), phenylmethylsulfonyl fluoride (PMSF), trichoroacetic-acid (TCA), protease inhibitor cocktail (P8849), were purchased from Sigma.

\section{Whole exome sequencing (WES) analysis}

Proband's genomic DNA (isolated from peripheral blood leukocytes) was captured using a SureSelect Human All Exon V7 (Agilent Technologies) exome enrichment kit and paired-end 150-nt sequencing was carried out on an Illumina NextSeq 550 platform (Illumina). Data analysis was conducted using a pipeline developed in the Institute of Mother and Child in Warsaw, Poland. Briefly, the BWA MEM (Burrow-Wheeler Aligner) was used to align reads to the reference genome GRCh38, single nucleotide variants and small insertion/deletions were detected using Genome Analysis Toolkit 4, Haplotype-Caller was used for variant calling and Ensembl Variant Effect Predictor for annotation. Variant status was further evaluated using the population (GnomAD v.3.0) and disease databases (ClinVar and Human Gene Mutation Database) and prediction tools available at VarSome website (https://varsome.com). Variant classification was performed in accordance with the American College of Medical Genetics (ACMG) recommendations [27]. Patient parents signed the informed consent for genetic analysis.

\section{Sanger sequencing}

Sanger sequencing was used as a confirmatory test in proband and in his parents for confirmation of biparental origin of the variant. The primers were self-designed using PRIMER3 software. Primers sequences available on request. Fluorograms were analysed using Mutation Surveyor v5.0.1 (Softgenetics).

\section{Biochemical analysis in cerebrospinal fluid (CSF), in urine and in plasma}

Collection and handling of CSF samples were performed strictly following a standardized procedure on the basis of former established methods [28]. Reversed phase HPLC and electrochemical detection was used for measurement of biogenic amine metabolites; organic acids profile in urine was investigated by gas chromatographic with mass spectrometric separation after trimethylsilylation for the semi-quantitative determination of vanillactic acid (VLA) and acetyl vanilalanine (AVA) concentrations. Reference range for CSF biogenic amine metabolites were obtained using CSF 
samples taken from patients with neurological disorders without extrapyramidal seizures. The agedependent metabolite concentration and an appropriately selected control group were used for comparison with the values obtain from our patient.

AADC enzyme activity in serum was measured as previously described [28, 29].

\section{Site-directed mutagenesis, expression and purification of P330L AADC}

WT AADC and P330L variant were obtained as previously described [23]. Mutagenesis reaction was performed using the Quick-Change II kit (Agilent technologies) using the oligonucleotide CCTTTAGACTGGACCTCACTTACCTGAAGC and its complement. All mutations were confirmed by DNA sequence analysis of the whole ORF.

WT AADC and P330L variant were expressed and purified as described in [23]. The enzymes concentration was determined using an $\varepsilon_{\mathrm{M}}$ of $1.42 \cdot 10^{5} \mathrm{M}^{-1} \mathrm{~cm}^{-1}$ at $280 \mathrm{~nm}$. PLP content was determined by releasing the coenzyme in $0.1 \mathrm{M} \mathrm{NaOH}$ using $\varepsilon_{\mathrm{M}}$ of $6600 \mathrm{M}^{-1} \mathrm{~cm}^{-1}$ at $388 \mathrm{~nm}$ [30].

\section{Apoenzyme preparation and coenzyme binding affinity measurements}

Apoenzyme was obtained by incubating $5 \mu \mathrm{M}$ holoenzyme with $15 \mathrm{mM}$ hydroxylamine in $0.5 \mathrm{M}$ potassium phosphate buffer $\mathrm{pH} 6.8$ at room temperature overnight and loaded on a Desalting 26/10 column (GE Healthcare) preequilibrated with $0.5 \mathrm{M}$ potassium phosphate buffer $\mathrm{pH} 6.8$ and eluted at $1 \mathrm{~mL} / \mathrm{min}$. The eluted enzyme was then concentrated on an Amicon Ultra 15 concentrators (Millipore) and washed with $100 \mathrm{mM}$ potassium phosphate buffer $\mathrm{pH}$ 7.4. The equilibrium apparent dissociation constant for PLP, $\mathrm{K}_{\mathrm{D}(\mathrm{PLP})}$, was determined as described in [23].

The data were fitted to the following equation (1):

$Y=Y_{M A X} \frac{[E] t+[P L P] t+K D(P L P)-\sqrt{([E] t+[P L P] t+K D(P L P)) 2-4[E] t[P L P] t}}{2[E] t}$

where [E]t and [PLP]t are the total concentrations of the enzyme and PLP, respectively, Y refers to the intrinsic quenching changes at a PLP concentration, and Ymax refers to the fluorescence changes when all enzyme molecules are complexed with coenzyme. Curves fitting was performed using Prism, 8.4.0, (GraphPad).

\section{Kinetic parameters, dopamine detection and coenzyme content determination at different pH}

To determine kinetic parameters of the decarboxylase reaction, AADC and P330L variant were incubated respectively at $5 \mathrm{nM}$ and $3 \mu \mathrm{M}$ final concentration in presence of $1 \mu \mathrm{M}$ and $100 \mu \mathrm{M}$ exogenous PLP and appropriate different L-Dopa and L-5HTP concentrations. The final reaction 
volume was $225 \mu \mathrm{L}$ in $100 \mathrm{mM}$ potassium phosphate buffer $\mathrm{pH}$ 7.4. Reaction times were set in order to detect a linear product formation. The mixtures were then quenched with $25 \mu \mathrm{L}$ of a $100 \%$ TCA solution. Proteins were precipitated in ice and removed by centrifugation. Supernatants were analyzed by HPLC as described [31, 32] using a Gemini C18 column (150 A, $4.6 \mathrm{~mm}$, Phenomenex, CA, USA) on a Jasco PU-2080 Plus HPLC system equipped with a UV-1570 detector set at $295 \mathrm{~nm}$. Samples were eluted in $100 \mathrm{mM}$ potassium phosphate, $\mathrm{pH} 2.35$, at a flow rate of $1 \mathrm{~mL} / \mathrm{min}$. Standard curves of dopamine peak area were prepared with commercially available dopamine. To determine the kinetic parameters as a function of $\mathrm{pH}$, the buffer was $50 \mathrm{mM}$ bis-tris-propane in order to avoid ionic strength effects. Data were fitted to the appropriate equation (2) using Prism, 8.4.0, (GraphPad).

$\log y=\log \frac{C}{1+H / K a}$

where y represents either $k_{\text {cat }}$ or $k_{\text {cat }} / \mathrm{K}_{\mathrm{m}}, \mathrm{C}$ is the $\mathrm{pH}$ independent value $\mathrm{K}_{\mathrm{a}}$ the ionization constant. To determine P330L coenzyme content in the presence of different substrates (2 mM L-Dopa and 2 mM L-5HTP) or P330L and WT coenzyme content with $2 \mathrm{mM} \mathrm{DME}$, the enzymatic species were incubated with the appropriate substrate/ligand at $10 \mu \mathrm{M}$ protein concentration with in $100 \mathrm{mM}$ potassium phosphate buffer, $\mathrm{pH} 7.4$ at $25^{\circ} \mathrm{C}$. The same analysis was performed for P330L in bis-trispropane $50 \mathrm{mM}$ buffer at $\mathrm{pH} 6.5,7.5$ and 8.5. HPLC detection was carried out as above. Standard curves of peak area as a function of coenzyme or cyclic adducts concentration were prepared with commercially available PLP and Pictet-Spengler adduct was obtained by incubating PLP with LDopa, L-5HTP and DME, as already described [23].

\section{Spectroscopic measurements}

All spectral measurements were acquired in $100 \mathrm{mM}$ potassium phosphate, $\mathrm{pH} 7.4$, at $25^{\circ} \mathrm{C}$ except for far-UV CD spectra which were recorded in $30 \mathrm{mM}$ potassium phosphate, $\mathrm{pH}$ 7.4. CD measurements were recorded with a Jasco J-710 spectropolarimeter at a scan speed of $50 \mathrm{~nm} / \mathrm{min}$ with a bandwidth of $2 \mathrm{~nm}$ at a protein concentration of $1-5 \mu \mathrm{M}$. Thermal denaturation was performed by monitoring the $\mathrm{CD}$ signal at $222 \mathrm{~nm}$ of $4 \mu \mathrm{M}$ enzyme on a $25-90{ }^{\circ} \mathrm{C}$ linear temperature gradient, with a temperature slope of $1{ }^{\circ} \mathrm{C} / \mathrm{min} .100 \mu \mathrm{M}$ of exogenous PLP was added to the holoenzymes. Analysis of the external aldimine spectral modifications were carried out on a Jasco V-550 spectrophotometer at a protein concentration of $10 \mu \mathrm{M}$ in absence or in presence of saturating concentration of substrates L-Dopa and L-5HTP. Fluorescence emission spectra were recorded on a Jasco FP-8500 fluorimeter. Internal aldimine of AADC species as function of $\mathrm{pH}$ values was carriedout at a protein concentration of $10 \mu \mathrm{M}$ in $50 \mathrm{mM}$ Bis-Tris-propane buffer over the $\mathrm{pH}$ range $6.0-$ 9.0. Absorbance data were fitted to equations (3) and (4): 


$$
\begin{aligned}
& A=\frac{A 1-A 2}{1+10^{\text {pH-pKspec }}}+A 2 \\
& A=\frac{A 1-A 2}{1+10^{\text {pspec }-p H}}+A 2
\end{aligned}
$$

where $A 1$ and $A 2$ are the higher and the lower absorbance limits at a particular wavelength.

\section{Dynamic light scattering (DLS) measurements}

DLS measurements were obtained on a Zetasizer Nano ZS instrument (Malvern), using disposable ZEN0112 polystyrene cuvettes. Setting used for particle size measurements was the following: solvent refractive index 1.330 , viscosity $0.8872 \mathrm{cP}$, protein refractive index 1.450 , protein absorption 0.001 , temperature $25^{\circ} \mathrm{C}$, equilibration 2 min, measurement angle $173 \AA$ A backscatter, analysis model multiple narrow modes. Samples were prepared at 3-4 $\mu \mathrm{M}$ protein concentration in $100 \mathrm{mM}$ potassium phosphate, $\mathrm{pH}$ 7.4. Native holo forms were preincubated for 30 minutes with additional $100 \mu \mathrm{M}$ PLP and $2 \mathrm{mM}$ cDopa bound species for 30 minutes before analysis. All samples were filtered using a $0.02 \mu \mathrm{m}$ Anotop 10 filter (Whatman). The reported sizes are based on 40 measurements, each consisting of 12-18 runs. Particle size for each sample is indicated as mean \pm standard deviation (SD). The results were compared using Student's t-test: ***P $<0.001 ; * * * * \mathrm{P}<0.0001$.

\section{Limited proteolysis experiments}

Limited proteolysis was performed by incubating $0.54 \mathrm{mg} / \mathrm{mL}$ AADC (both WT and P330L) with trypsin at the final E/S ratio of 1:100 (w/w) in $100 \mathrm{mM}$ potassium phosphate, $\mathrm{pH} 7.4$ at $25^{\circ} \mathrm{C}$, with the addition of $2 \mathrm{mM}$ DME. Aliquots were withdrawn at different times and compared with AADC sample in absence of trypsin. At each time point, the reaction was boiled in SDS sample buffer to stop trypsin activity. The digestion products were separated by SDS/PAGE, and gels were stained with Coomassie.

\section{Bioinformatic analyses}

Human AADC sequence (P20711) was aligned with human group II decarboxylase sequences (HDC: P19113, CSAD: Q9Y600, GAD65: Q05329, GAD67: Q99259) retrieved from UniProt database and aligned using Multiple Sequence Alignment software CLUSTALW OMEGA on EMBL-EBI server (http://www.ebi.ac.uk/). 
The position and possible interactions of Pro330 as well as the modifications induced by the P330L substitution have been analyzed using two approaches. First, we started the homology modeling performed with the Yasara Structure package [http://www.yasara.org/]. Three template structures accessible in PDB (1js3 pig kidney AADC bound to the inhibitor cDopa, 1js6 pig kidney AADC and 4 elo human histidine decarboxylase bound to histidine methylester) were identified automatically. The regions absent in the template structures of pig AADC, including residues 328-339 of the CL, were modelled using knowledge-based loop modeler implemented in the Yasara package. The final hybrid model was built from the best single-template model, in which every sub-optimal region was iteratively replaced with analogous fragments adopted from alternative models. The thermodynamic effect of single-point replacement Pro330Leu was then assessed with the aid of the FoldX program [http://foldxsuite.crg.eu/], independently for the highest-scored single-template models with open or closed conformation of the CL loop.

Pro to Leu substitution was obtained and minimized using the Protein Preparation Wizard [33] in the program BioLuminate within Schrödinger Suite 2021-4 and applying the OPLS4 force field (BioLuminate, Schrödinger, LLC, New York, NY, 2021). Structural visualization, measurements and figures of the human holoAADC structure were carried out using Pymol 2.2.3 (The PyMOL Molecular Graphics 50 System, Version 2.0 Schrödinger, LLC, New York, NY, 2021). Frustration analysis of the CL was performed by the Frustatometer server [34] for the obtained WT and P330L holoAADC in both the open and closed CL conformations.

\section{Results and Discussion}

\section{Clinical phenotype of a patient carrying the homozygous P330L substitution in DDC gene}

A currently 2.5-year-old boy from healthy consanguineous parents, with uneventful pre-and perinatal period and family history was born on time with normal body weight, length and head circumference, and received 10 points in Apgar scale. Since infancy he was unable to suck, and recurrent vomiting with excessive salivation were observed. Since the age of 8 months, he suffered from episodes of anxiety, crying and increased sweating have been observed. Since the second year a generalized weakness had appeared. On pyridoxine $50 \mathrm{mg} / \mathrm{d}$ and then $150 \mathrm{mg} / \mathrm{d}$ the patient was quite stable. But at age of 2 years old he suffered from SARS-COV2 infection, and oculogyric crises appeared. Folinate had been introduced with initially clinical improvement - he became more active and stronger, with better sleep. Actually, he suffers from marked hypotonia and psychomotor development retardation, but mainly in gross motor skills. Further reduction of the patient's overall activity and muscle tone is observed in the end of the day with evident improvement after sleeping. 
Additional analyses of MRI and EEG showed mild, non-specific abnormalities.

\section{Diagnostic laboratory tests of CSF, urine and plasma of the patient}

The CSF biogenic amine metabolites analysis showed reduced concentrations of homovanillic acid (HVA) $133 \mathrm{nmol} / \mathrm{L}$ (236-645 nmol/L) and 5-OH-indolacetic acid (5-HIAA) $10 \mathrm{nmol} / \mathrm{L}$ (97-367 $\mathrm{nmol} / \mathrm{L})$, and an increased concentration of 3-ortho methyl dopa (3-OMD) of $1029 \mathrm{nmol} / \mathrm{L}$ (0-50 $\mathrm{nmol} / \mathrm{L})$ and L-5HTP $133 \mathrm{nmol} / \mathrm{L}(0-25 \mathrm{nmol} / \mathrm{L})$. The CSF 5-methyltetrahydrofolate (5-HTF) concentration was normal $200 \mathrm{nmol} / \mathrm{L}(78-216 \mathrm{nmol} / \mathrm{L})$ before folinate treatment. Curiously, HVA is relatively high. Interestingly, even if decreased, the HVA level is relatively high with respect to the other measured markers. The urine metabolites of dopamine precursors VLA and AVA were strongly increased in urine organic acid profile; this metabolite pattern is fully characteristic of an AADC deficiency.

The AADC activity in the plasma using L-Dopa as a substrate was $1.0 \mathrm{mU} / \mathrm{L}$ (reference range is 16$99 \mathrm{mU} / \mathrm{L}$ ) and using L-5HTP it was $0.06 \mathrm{mU} / \mathrm{L}$ (reference range is $1.0-7.1 \mathrm{mU} / \mathrm{L}$ ). Thus, AADC enzymatic activity was severely impaired for both substrates.

\section{Genetic results}

The proband's WES analysis revealed the presence of DDC gene variant NM_001082971.2:c.989C>T (p.Pro330Leu) in 74 of 77 reads, indicating its homozygosity, which was further confirmed by Sanger sequencing (Fig. S1A). Biparental origin of the variant was confirmed by Sanger sequencing of the parental DNA (Fig S1B). The variant was not reported previously according to GnomAD, ClinVar and HGMD databases. In the ACMG classification was initially assigned as likely pathogenic, which later, after the completion of in vitro studies, was changed into pathogenic.

\section{Sequence comparison, modelling and frustration index of the CL for AADC reveal that Pro330 concurs to the functional flexibility of the $C L$}

The sequence alignment of the CL region of human Group II $\alpha$-decarboxylases (Fig. S2) shows that Pro330 is conserved in AADC and HDC or substituted by an alanine residue in CSAD and in the two GAD isoforms, highlighting that a small aliphatic residue is requested in that position. A model of the loop of AADC was already built from the coordinates of the solved pig enzyme modelling the solved loop region of the homologous human HDC [14]. The active site organization with the relevant residues playing a role in the PLP binding cleft and substrate aromatic side chain positioning have 
been already identified [2]. The CL is presumed to be highly flexible, and a Pro330 residue could serve as a hinge and thus dictate the freedom degrees of the CL to position the catalytic Tyr332 [12]. Starting from the pig AADC structure and HDC loop region, we obtained a few possible CL conformations where Pro330 approaches the active site of the opposite monomer, as already suggested $[6,12,14]$. Although this loop seems to adopt the closed conformation in the highestscored model, also open conformations are observed in some others. In general, all models based on native holo pig AADC (PDB 1js6) are in the open conformation, based on HDC in complex with the ligand histidine methyl ester (PDB 4e1o) are in the closed conformation, while those based on cDopabound holo pig AADC (PDB 1js3) are either in open or in closed form. These two alternative models, the quality of which was assessed as "good", are shown in Fig. 1A. In particular, Pro330 is placed inside the active site of the opposite subunit when the CL is in the closed conformation, while it is solvent exposed when the CL is open. The thermodynamic effect of the replacement of Pro330 with Leu was assessed for the two highest-scored models, one with both loops in the open conformation and the other with both in the closed form. In both CL conformational states, the P330L replacement was found unfavorable. However, the closed state is distorted much more $(2.5 \mathrm{kcal} / \mathrm{mol})$ than the open one $(0.5 \mathrm{kcal} / \mathrm{mol})$. Thus, upon P330L replacement, the open state of the protein is less disturbed. The difference of about $2 \mathrm{kcal} / \mathrm{mol}$ corresponds roughly to a 30 -fold shift of the conformational equilibrium towards the CL open state.

Following this view, the P330L replacement is expected to affect the positioning of the loop.

A deep inspection reveals that Pro330 represents a conformational constraint that allows the CL to bend and properly position Tyr332 bridging it to both His192' and Ser193' (the prime denotes residues of the neighboring subunit) (Fig. 1B). The bending is reinforced by a number of H-bonds concurring in holding in place the loop: Arg347 is involved in a H-bonding network with Asp345 and His335. On the opposite side, the CL is maintained into the active site groove by interactions with a structural motif (a beta sheet, residues 436-441) belonging to the CTD distant 4-5 $\AA$ from the flexible stretch, mainly by Thr331 reaching His439'. It is reasonable to propose that the substitution of Pro330 with Leu would lead to a loosening of the conformational role played by Pro and although the chemical nature of the residue is not altered, its conformational freedom could be affected, with possible consequences on Tyr332 proper positioning. In order to gain some insight into the setting/twisting of the unstructured CL into the opposite active site, we computed the frustration index of residue in the Pro330 and Leu330 AADC proteins. The frustration notion, arising from statistical physics and applied to biomolecules [35], is suitable to evaluate the conformational "freedom" of highly flexible regions [36]. The single residue frustration index of Pro330 is below -1 only for one monomer in the closed CL state (Table 1) and in the other cases it is neutrally frustrated. A substituted 
Leu in position 330 increases the frustration index thus increasing the energetically favorable interactions for both the subunits in the open and closed CL state. This could mean that the mutated residue has no gross energetical disadvantage in itself. Then, we calculated the mutational and conformational frustration index of the contact pairs involving the residue at position 330 and its related network (Fig. 1C and D). The total number of highly frustrated interactions increases in mutational frustration when Leu is present at the place of Pro330 in the closed CL state. In configurational frustration, the number of highly frustrated interactions between WT and P330L variant is equal when the $\mathrm{CL}$ is in the closed state and decreases in the P330L with respect to the WT by one unfavorable interaction in the CL open state. Thus, the Pro-to-Leu substitution leads to a less frustrated open CL.

Overall, the in silico analysis indicates the importance of Pro330 in keeping the CL well fitted to orient the catalytic residue Tyr332 properly.

Interestingly, the dimer hydrodynamic radius of apoWT presents a small but statistically significant $(\mathrm{p}<0.0001)$ increase with respect to the holoWT and its cDopa bound form (Fig. 2 and Table 2). A similar slight difference is observed comparing holoAADC in the absence or presence of cDopa. It is not unprecedent that a small difference in compactness could give rise to small changes in this parameter, as in HDC whose oxidized more compact form is slightly smaller than the reduced one [37] due to the presence of only one additional disulfide bridge. Meanwhile, apoP330L dimer diameter is slightly increased with respect to apoWT $(\mathrm{p}<0.0001)$ and it is not significantly different for the forms of the variant (apo, holo, and cDopa-bound). Notably, cDopa binding does not lead to a more compact structure in case of holoP330L. This reinforces the fact that a ligand bound to PLP in the active site of the P330L variant does not induce a conformational shift as in the corresponding WT species.

The structural features of P330L AADC are not affected by amino acid substitution but CL flexibility is compromised

The far UV CD spectra of apo and holo P330L show a similar profile and secondary structure content and are not different from the corresponding ones of the WT suggesting that the overall fold is not affected by the amino acid substitution (Fig. 3A). This is also mirrored by similar values of thermostability at $222 \mathrm{~nm}$ (Table 3). Moreover, intrinsic fluorescence (Fig. 3B) and near UV (Fig. 3C) spectra of P330L of both holo and apo enzymes are well superimposable to those of the WT, suggesting that the amino acid alteration on the highly mobile CL element has not determined changes in terms of tertiary structure in the protein. Notably, the visible CD (Fig. 3C) and absorbance spectra (inset Fig. 3C) of P330L AADC present the same signals associated to the tautomeric equilibrium of 
PLP bound to the active site Lys303 in forms of enolimine and ketoenamine tautomers as the WT [38] suggesting that the PLP binding mode is not altered by substitution. However, while the fluorescence emission of the ketoenamine tautomer of P330L is identical to that of the WT $\left(\lambda_{\max }=\right.$ $513 \mathrm{~nm}$ ), the emission signal of the P330L enolimine fluorophore displays a smaller quantum yield (about $70 \%$ ) with an identical emission maximum $\left(\lambda_{\mathrm{em}}=387 \mathrm{~nm}\right)$ concomitant with a small increased contribution of the $513 \mathrm{~nm}$ emission signal indicating a subtle dissimilar microenvironment such as a change in the PLP microenvironment [39].

However, this has no consequence on the PLP equilibrium dissociation constant that results $123 \pm 19$ $\mathrm{nM}$, a value almost identical to that of the WT $(101 \pm 10 \mathrm{nM})$ measured under the same experimental conditions.

Given the proximity of the substituted amino acid to the tryptic site (Lys334-His335 bond) of the WT enzyme [9], we have determined the trypsin accessibility to P330L with respect to the WT in the unliganded form or complexed with DME, the esterified form of L-Dopa unable to undergo decarboxylation [14]. While both unliganded WT and P330L show a rate of proteolysis of about $0.082 \mathrm{~min}^{-1}$ under the same experimental conditions, DME-P330L proteolysis slightly slows down $\left(0.036 \mathrm{~min}^{-1}\right)$. WT-DME, instead, shows a marked protection from digestion (Fig. 4A and B), thus suggesting that a Leu residue in that position does not dramatically alter CL exposure in the absence of a ligand, but eventually exerts its effect on CL flexibility, possibly hindering loop closure upon the active site. In this sense, limited proteolysis data suggest that the task of Pro330 is to concur in governing CL flexibility.

\section{The kinetic competence of $\mathrm{P330L}$ is highly compromised}

When P330L AADC variant was incubated with both substrates L-Dopa and L-5HTP (for details see under the Materials and Methods section) a drop in the catalytic activity is observed. Table 4 shows the kinetic parameters and evidences how the highly affected activity versus L-Dopa $(0.15 \%$ in terms of catalytic efficiency) seems to be driven mainly by a decrease in $k_{\text {cat }}(0.5 \%$ that of the WT) while $\mathrm{K}_{\mathrm{m}}$ increases by 3.5 -fold that of the WT. A similar behavior is seen with L-5HTP with $k_{\text {cat }}$ resulting $1.6 \%$ that of the WT, while $\mathrm{K}_{\mathrm{m}}$ is increased of 3.2-fold leading to a catalytic efficiency of $0.5 \%$ that of the WT. Thus, the P330L substitution compromises catalysis rather than overall protein structural features.

In order to unravel the molecular reason for the loss of catalytic competence we carried out a spectral analysis of coenzyme modification upon substrate binding. The addition of $2 \mathrm{mM}$ L-Dopa to $10 \mu \mathrm{M}$ P330L AADC in potassium phosphate buffer $\mathrm{pH} 7.4$ at $25^{\circ} \mathrm{C}$ determines an immediate increase at $420 \mathrm{~nm}$ that slowly decreases with time concomitant with an increase at about $328 \mathrm{~nm}$ (Fig. 5A). 
Interestingly, the WT at the same $\mathrm{pH}$ value shows different spectral signals with a $420 \mathrm{~nm}$ band converting into a $390 \mathrm{~nm}$ absorbing species attributed to a more reactive external aldimine form [40, 41] (see below). HPLC analysis of the reaction mixture reveals that about $20 \%$ of the coenzyme decreases (Fig. 5B), being converted into 15\% of pyridoxamine 5'-phosphate (PMP) (arising from the ability of AADC to catalyze multiple side reactions in addition to the main decarboxylation one [42-45], as evidenced in other AADC variants [23]) and 5\% of the Pictet-Spengler adduct, a cyclic compound formed by spontaneous non-enzymatic nucleophilic addition of the aromatic ring of the aromatic substrate to the nitrogen atom of the external aldimine complex [9]. The formation of this compound depletes the original PLP content and leads to enzyme inactivation. Its recovery is a hallmark of coenzyme exposure to the solvent and has been already detected in several AADC deficiency variants [22, 23], especially when compromised catalysis is due to structural disassembling. This is not the case for P330L that, as other so-called catalytic variants such as L353P [23], forms very low amounts of cyclic compound not responsible for the measured drop of activity. The addition of $2 \mathrm{mM} \mathrm{L-5HTP}$ to $10 \mu \mathrm{M}$ P330L AADC in potassium phosphate buffer $\mathrm{pH} 7.4$ at $25^{\circ} \mathrm{C}$ leads to a slow increase at $420 \mathrm{~nm}$ that continues to increase with time (Fig. 5C). The HPLC analysis shows that PLP slowly decreases, being converted into PLP-L-5HTP cyclic adduct, and serotonin linearly increases (Fig.5D).

Altogether, the marked decrease in catalytic efficiency of P330L is not driven by a gross protein structural change nor by an opening of the active site, since the cyclic adduct formation (signature of PLP exposure to the solvent) is kept at negligeable levels.

\section{The substitution of Pro330 with Leu affects the conformation of the internal aldimine that triggers a dropped kinetic capability of the external aldimine}

Up to now, the only clue indicating an alteration at the active site microenvironment triggered by the Pro-to-Leu substitution is the anomalous behavior of the enolimine tautomer of the internal aldimine and the absence of the external aldimine with L-Dopa absorbing at $390 \mathrm{~nm}$ typical of the WT and associated to more efficient catalysis [40,41]. Firstly, we determined the $\mathrm{pH}$ dependency of the internal aldimine absorbance bands to investigate tautomeric equilibrium. The equilibrium among the ketoenamine and the enolimine tautomers of WT AADC has been already determined and attributed to a residue with pKa of about 7.3 controlling it [41]. Interestingly, the equilibrium distribution of tautomers of the internal aldimine of $\mathrm{P} 330 \mathrm{~L}$ as a function of $\mathrm{pH}$ shows that the ketoenamine absorbance band displays a pKa of $8.2 \pm 0.2$, while the enolimine absorbance signal seems to be mostly insensitive of pH (Fig. 6A and inset). If the fluorescence emission of P330L is evaluated upon excitation at $420 \mathrm{~nm}$ as a function of $\mathrm{pH}$, the emission of the ketoenamine at $513 \mathrm{~nm}$ shows a 
$\mathrm{pH}$ dependence with a measured $\mathrm{pKa}(8.5 \pm 0.2)$ that overlaps to the value obtained in absorbance. Curiously, if excitation is set at $335 \mathrm{~nm}$, the $387 \mathrm{~nm}$ emission is $\mathrm{pH}$ dependent with a pKa of $8.3 \pm$ 0.1 (Fig. 6B and inset). The responsivity of this species in fluorescence with respect to absorbance could be ascribed to the more sensitive spectrofluorimetric detection. The different $\mathrm{pKa}$ value of the residue/s controlling the internal aldimine tautomeric equilibrium in $\mathrm{P} 330 \mathrm{~L}$ with respect to the WT could be a consequence of CL misplacing and should be mirrored by some alteration in the catalytic external aldimine intermediate when the substrates bind the coenzyme and start the catalytic process. Interestingly, the P330L variant in the presence of L-Dopa forms a $420 \mathrm{~nm}$ absorbing band (not converting into a $390 \mathrm{~nm}$ species over the entire $\mathrm{pH}$ range as, instead, the WT accumulates [40, 41]) that decreases with time concomitant with an increase at $328 \mathrm{~nm}$ (Fig. S3). By loading onto HPLC the reaction mixtures at $\mathrm{pH} 6.5,7.5$ and 8.5 it can be observed that dopamine is linearly formed during 1 hour with concomitant small conversion of the PLP cofactor into PMP and Pictet-Spengler adduct, that globally deplete less than $20 \%$ of the total coenzyme (Fig. S3). Thus, these coenzymatic species are not responsible for low activity of P330L.

The anomalous catalytic behavior of P330L AADC is also confirmed by the dependence of the catalytic parameters on $\mathrm{pH}$. Both $\log \mathrm{k}_{\text {cat }}$ and $\log \mathrm{k}_{\mathrm{cat}} / \mathrm{K}_{\mathrm{m}}$ plot increase as a function of $\mathrm{pH}$ with a $\mathrm{pKa}$ of $6.9 \pm 0.1$ and $7.0 \pm 0.2$, respectively (Fig. 6C and D). Since this pKa value is present in both plots, it can be assigned to a residue (probably the same) essential for catalysis. Interestingly, this $\mathrm{pH}$ dependence is rather different from that exhibited by the WT enzyme where the acidic pKa value on the $\log k_{c a t}$ and $\log k_{c a t} / \mathrm{K}_{\mathrm{m}}$ plots (pKa around 6.3) was attributed to the 4-N'-deprotonation of the external aldimine leading to the $390 \mathrm{~nm}$ species [41]. Since in P330L the $390 \mathrm{~nm}$ external aldimine does not form, it is reasonable to attribute the pKa of 7 in P330L to a residue of the enzyme implicated in catalysis. The easiest attribution is His 192 implicated in deprotonating the catalytic Tyr332, as suggested $[38,45]$. The same could be proposed also to the pKa of about 6.3 of the WT AADC. The increase of $1 \mathrm{pH}$ unit mirrors that of the internal aldimine and should be a direct consequence of alteration of polarity of the microenvironment/catalytic intermediate positioning in the $\mathrm{P} 330 \mathrm{~L}$ variant. The absence of the $390 \mathrm{~nm}$ absorbing species that was attributed to an enolimine species [41, 45] more reactive than the $420 \mathrm{~nm}$ ketoenamine tautomer (that initially forms and is the only form present at acidic $\mathrm{pH}$ for the WT [41]) led us to consider if the binding mode of external aldimine is somehow altered in the P330L variant.

The addition to WT AADC of $2 \mathrm{mM}$ DME determines the appearance of two bands one absorbing at $398 \mathrm{~nm}$, indicative of the external aldimine intermediate, and the other at $328 \mathrm{~nm}$ [46]. While the 398 $\mathrm{nm}$ species remains unchanged with time, the $328 \mathrm{~nm}$ form increases (Fig. 7A). The spectral modifications could be attributed both to oxidation of the aromatic substrate and to a small amount 
of the Pictet-Spengler adduct produced, as the HPLC determination reveals (Fig. 7C). When $2 \mathrm{mM}$ DME is added to $10 \mu \mathrm{M}$ P330L along with a band absorbing at $393 \mathrm{~nm}$ and another at $325 \mathrm{~nm}$, another band at $500 \mathrm{~nm}$ appears attributable to a quinonoid species as for other variants belonging to the same loop 3 region [14] (Fig. 7B). The HPLC analysis of the reaction mixture evidences a slight propensity for P330L to produce the irreversible cyclic adduct, which is almost absent in the WT (Fig. 7C). This behavior is reminiscent of that of the nicked AADC [9], a species cleaved by trypsin (as reported above) between residues Lys334-His335 on the CL, unable to perform decarboxylation but able to bind and perform other reactions on some aromatic amino acids and or amines or derivatives [45]. In particular, nicked AADC is able to bind DME leading to the formation of a quinonoid species at 500 $\mathrm{nm}$ [46]. This was interpreted as a mispositioning of the external aldimine. Here, we can add that each time the CL is somehow displaced by an alteration of the H-bonding network, this leads to catalytic inability enhancing the inherent propensity of PLP to react non enzymatically with its aromatic substrates/analogs.

\section{Conclusions}

The molecular defect of the novel P330L variant causing AADC deficiency and severe symptoms to the patient can be ascribed to the alteration of the conformational equilibrium between the open and the closed form of the CL, essential for enzymatic function. In the closed catalytically competent conformation of the WT enzyme, Pro330 is conveniently positioned by a broad network of interactions (weak electrostatic and H-bonds) promoted by the interaction of some residues such as Asp329, Asp345, Arg347, His348 of both extremities of loop3 (residues 323-357) to residues 330339 of the CL and to some elements of the LD (His192 and Ser193) and of the CTD (His439) as shown in Fig. 1B. In this manner, the cavity of the active site accommodates the CL that is free to enter (closed conformation) and exit (open conformation). In this context, Pro330 seems to act as pivot to allow the CL (and thus Tyr332) to be properly inserted into the active site once the external aldimine is formed, as shown by molecular modelling analyses and protection by proteolysis. The substitution with Leu on one hand destroys the H-bonding network rendering the CL less able to sit in the closed state, on the other it influences internal and external aldimine placing, thus affecting catalysis rather than global protein structure. The anomalous polarity and conformation of the internal aldimine, as fluorescence and $\mathrm{pH}$ data suggest, are reflected into the external aldimine mispositioning revealed by the absence of the $390 \mathrm{~nm}$ band at any $\mathrm{pH}$ value in the presence of L-Dopa and the accumulation of a quinonoid species in the presence of the analog DME. The latter is also formed in other AADC pathogenic variants of the loop3 region [14]. This behavior has been proposed to be due to alteration of the H-bonding network governing the positioning of the catalytic residue Tyr332. The 
great decrease in catalytic efficiency for both substrates does not seem to be due to Pictet-Spengler adduct formation (that would have been indicative of a completely exposed active site [9]) rather to an impaired conformation of the external aldimine detrimental for efficient catalysis. In addition, both the PLP microenvironment and the positioning of the catalytic intermediate evidence alterations in the chemistry of some residues, as witnessed by the different $\mathrm{pKa}$ values of both the internal aldimine and of the catalytic parameters with respect to the WT. This pathogenic amino acidic substitution has been so deeply investigated to unravel the molecular basis for AADC deficiency given the fact that it is mapped on an essential structural and functional element. Recently, a promising precision therapy has been proposed for an AADC deficiency patient by correlation of bioinformatics, biochemical and in solution experiments together with iPSCs validation [47]. Given the severe catalytic impairment exhibited by this novel AADC protein variant, the therapeutic suggestion is to sustain the ongoing clinical management with a dopamine (and serotonin) agonist, in order to enhance the extremely low endogenous activity. 


\section{Tables}

Table 1-Single-residue, mutational and configurational frustration level index of Pro330 (in the WT AADC) and Leu330 (in the P330L AADC variant) in the open and closed conformation of the CL for the two monomers of AADC

\begin{tabular}{|c|c|c|c|}
\hline & $\begin{array}{l}\text { single- } \\
\text { residue }\end{array}$ & mutational $^{\mathrm{a}}$ & configurational $^{\mathbf{a}}$ \\
\hline WT & & & -1.988 (residues 328'-330’) \\
\hline CL open & & -- & -1.62 (residues 329'-331') \\
\hline Monomer A & -1.036 & & -1.643 (residues 331'-333') \\
\hline Monomer B & -0.719 & & -2.714 (residues $331^{\prime}-334^{\prime}$ ) \\
\hline CL closed & & -1.114 (residues 193-330’) & -2.534 (residues 329'-333') \\
\hline Monomer A & -0.928 & & -1.199 (residues 329'-334') \\
\hline \multirow[t]{2}{*}{ Monomer B } & -0.702 & & -1.686 (residues 331'-334') \\
\hline & & & -1.118 (residues $332^{\prime}-334^{\prime}$ ) \\
\hline P330L & & & -1.662 (residues 329'-331') \\
\hline CL open & & -- & -1.682 (residues $\left.331^{\prime}-333^{\prime}\right)$ \\
\hline Monomer A & 1.188 & & -2.722 (residues $331^{\prime}-334^{\prime}$ ) \\
\hline Monomer B & 0.912 & & \\
\hline CL closed & & -1.248 (residues 329'-334') & -2.459 (residues 329'-333') \\
\hline Monomer A & 1.162 & -1.149 (residues 331'-334') & -1.181 (residues 329'-334') \\
\hline \multirow[t]{2}{*}{ Monomer B } & 0.912 & & -1.668 (residues 331'-334') \\
\hline & & & -1.102 (residues 332'-334') \\
\hline
\end{tabular}

athe prime (') denotes residues from the neighboring subunit

Table 2-Hydrodynamic radius of apo, holo and cDopa bound WT and P330L AADC in $100 \mathrm{mM}$ potassium phosphate, $\mathrm{pH} 7.4$

\begin{tabular}{lcccccc}
\hline Enzyme & \multicolumn{2}{c}{ WT } & \multicolumn{3}{c}{ P330L } \\
\hline Species & apo & holo & +cDopa & apo & holo & + cDopa \\
\hline Mean $(\mathrm{nm}) \pm \mathrm{SD}^{\mathrm{a}}$ & $9.63 \pm 0.34$ & $9.32 \pm 0.27$ & $9.14 \pm 0.15$ & $9.99 \pm 0.31$ & $9.83 \pm 0.53$ & $9.84 \pm 0.25$
\end{tabular}

${ }^{\mathrm{a}}$ Data are reported as mean \pm SD and expressed in (nm) 
Table 3-Thermostability at $222 \mathrm{~nm}$ of holo, apo and cDopa bound WT and P330L AADC in $100 \mathrm{mM}$ potassium phosphate buffer, $\mathrm{pH} 7.4$

\begin{tabular}{ccc}
\hline AADC & WT & P330L \\
\hline Apo & $62.6 \pm 0.2$ & $62.5 \pm 0.2$ \\
Holo & $68.5 \pm 0.2$ & $68.0 \pm 0.2$ \\
+ cDopa & $69.3 \pm 0.2$ & $69.3 \pm 0.2$ \\
\hline
\end{tabular}

Table 4-Kinetic parameters of WT and P330L AADC in $100 \mathrm{mM}$ potassium phosphate buffer, $\mathrm{pH}$ 7.4 at $25^{\circ} \mathrm{C}$.

\begin{tabular}{ccccc}
\hline Substrate & Enzyme & $k_{\text {cat }}\left(\mathrm{s}^{-1}\right)$ & $\mathrm{K}_{\mathrm{m}}(\mathrm{mM})$ & $k_{\text {cat }} / \mathrm{K}_{\mathrm{m}}\left(\mathrm{s}^{-1} \mathrm{mM}^{-1}\right)$ \\
\hline L-Dopa & WT & $5.5 \pm 0.1$ & $0.016 \pm 0.001$ & $344 \pm 22$ \\
& P330L & $0.0291 \pm 0.0004$ & $0.057 \pm 0.002$ & $0.51 \pm 0.05$ \\
& & & & \\
L-5HTP & WT & $0.56 \pm 0.02$ & $0.009 \pm 0.001$ & $62 \pm 7$ \\
& P330L & $0.0092 \pm 0.0003$ & $0.029 \pm 0.003$ & $0.32 \pm 0.03$
\end{tabular}




\section{Legends to figures}

Figure 1. Modelled catalytic loop (CL) of AADC. A) Dimeric AADC is shown as surface with the two monomers colored white and gray. PLP, cDopa, Pro330 and Tyr332 are shown as sticks. The modelled closed CL (cartoon tube, cyan) of one monomer is inserted into the active site of the other monomer with Pro330 and Tyr332 both in close proximity to PLP of the facing monomer, while the modelled open CL (cartoon tube, blue) is completely solvent exposed. B) The closed CL is favored by several interactions among residues of CL (in cyan), of loop3 (in cyan) and of the neighboring monomer (in yellow, the prime, ', denotes residues of the opposite subunit). Frustration of the CL in both the closed C) and open D) conformation calculated for the WT (monomer A showing the CL in cyan, monomer B in white, PLP in yellow) and P330L (monomer A showing the CL in blue, monomer $\mathrm{B}$ in white, PLP in yellow) AADC. Conformational and mutational highly frustrated pairs are represented as red links on the same figure for clarity. The analyses were performed using the Frustratometer server $[34,36]$ that does not include coenzymes and for this reason PLP has been shown for clarity. As above, residues belonging to the opposite monomer of the CL are indicated with the prime.

Figure 2. Hydrodynamic diameters calculated with DLS. Particle size relative to apo and holo AADC species as well as the protein bound to the inhibitor cDopa. Results were compared using Student's t test; $* * * \mathrm{P}<0.001 ; * * * * \mathrm{P}<0.0001$, ns: not significantly different.

Figure 3. Spectroscopic analysis of WT and P330L AADC species. HoloWT (black solid line), apoWT (black dashed line), holoP330L (blue solid line) and apoP330L (blue dashed line) AADC species were analyzed by means of spectroscopic techniques. A) Far-UV CD spectra were recorded at $1 \mu \mathrm{M}$ protein concentration in $30 \mathrm{mM}$ potassium-phosphate buffer, $\mathrm{pH} 7.4$ with the addition of 100 $\mu \mathrm{M}$ PLP for the holo forms. B) Intrinsic emission fluorescence recorded upon excitation at $280 \mathrm{~nm}$ in $100 \mathrm{mM}$ potassium-phosphate buffer solution at $\mathrm{pH} 7.4$ at $1 \mu \mathrm{M}$ protein concentration. C) NearUV CD spectra of apo and holo AADC species recorded at $5 \mu \mathrm{M}$ protein concentration in $100 \mathrm{mM}$ potassium-phosphate buffer $\mathrm{pH} 7.4$ with the addition of $100 \mu \mathrm{M}$ PLP for the holo forms. The inset shows the visible absorbance spectra of $10 \mu \mathrm{M}$ holo protein. D) Bound coenzyme emission fluorescence spectra of $1 \mu \mathrm{M}$ WT and P330L AADC recorded in $100 \mathrm{mM}$ potassium-phosphate buffer at pH 7.4 upon excitation at $335 \mathrm{~nm}$ (for enolimine emission) and $420 \mathrm{~nm}$ (for ketoenamine emission). 
Figure 4. Limited proteolysis of native and inhibitor bound AADC species. Limited proteolysis was carried out with a trypsin:AADC ratio of 1:100 (w/w) in presence of $100 \mu \mathrm{M}$ PLP alone or $2 \mathrm{mM}$ DME in $100 \mathrm{mM}$ potassium phosphate $\mathrm{pH} 7.4$ at $25^{\circ} \mathrm{C}$. At various time intervals, aliquots were withdrawn and subjected to SDS-PAGE. A) The lanes refer to the incubation times, MW= molecular weight markers. B) Time course of trypsin digestion of native AADC (black circles), native P330L (blue squares), both WT (grey circles) and P330L (light blue squares) in the presence of DME. Species are expressed as a percentage of initial undigested monomer.

Figure 5. Absorbance spectral changes and coenzyme content modification during the reaction of P330L AADC with L-Dopa and L-5HTP. All reactions were carried out in $100 \mathrm{mM}$ potassium phosphate, $\mathrm{pH} 7.4$ at $25^{\circ} \mathrm{C}$ for $1 \mathrm{~h}$. A) Spectral modification of $10 \mu \mathrm{M}$ P330L variant in presence of $2 \mathrm{mM}$ L-Dopa. The arrows indicate the increase at $328 \mathrm{~nm}$ and the concomitant decrease at $420 \mathrm{~nm}$ with time. B) HPLC analyses of the reaction mixture of $10 \mu \mathrm{M}$ P330L in presence of $2 \mathrm{mM}$ L-Dopa. PLP (grey circles), PLP-L-Dopa adduct (blue circles), PMP (green circles) and dopamine (orange circles). C) Spectral modification of $10 \mu \mathrm{M}$ P330L variant in presence of $2 \mathrm{mM}$ L-5HTP. The arrow indicates the increase at $425 \mathrm{~nm}$ with time. D) HPLC analyses results of the reaction mixture of 10 $\mu \mathrm{M}$ P330L and $2 \mathrm{mM}$ L-5HTP. PLP (grey circles), PLP-L-5HTP adduct (cyan circles), serotonin (orange circles). Dashed lines represent the absorbance spectra in absence of substrate.

Figure 6. pH dependence of the absorbance and fluorescence bands of P330L internal and WT and P330L external aldimine with DME and of the catalytic parameters for the P330L decarboxylation of L-Dopa. A) Absorbance spectra of $10 \mu \mathrm{M}$ P330L in $50 \mathrm{mM}$ bis-tris-propane buffer at different $\mathrm{pH}$ values. The inset shows the $\mathrm{pH}$ dependence of the enolimine (335 nm, circles)) and ketoenamine (420 nm, squares) tautomers. Solid lines represent the theoretical fitting according to eq. (3) and (4). B) Coenzyme emission fluorescence spectra of $1 \mu \mathrm{M}$ P330L in $50 \mathrm{mM}$ Bis-Trispropane buffer at different $\mathrm{pH}$ values upon excitation at $335 \mathrm{~nm}\left(\lambda_{\mathrm{em} \max }=387 \mathrm{~nm}\right)$ and $420 \mathrm{~nm}\left(\lambda_{\mathrm{em}}\right.$ $\max =513 \mathrm{~nm}$ ). The inset shows the theoretical fit of the experimental data of $\mathrm{pH}$ dependence of the enolimine (387 nm, circles) and ketoenamine (513 nm, squares) species. Solid lines represent the theoretical fitting according to eq. (3) and (4). C) $\log k_{\text {cat }}$ and D) $\log k_{\text {cat }} / \mathrm{K}_{\mathrm{m}}$ for the decarboxylation of L-Dopa by P330L AADC. The curves are obtained by fitting the experimental points to eq. (2). Experimental details are reported under the Materials and Methods section.

Figure 7. Absorbance spectra and HPLC analyses of the reaction of either WT or P330L AADC with DME. A) Absorbance spectra of $10 \mu \mathrm{M}$ WT AADC before (dashed line) and after the addition 
of $2 \mathrm{mM}$ DME (colored lines) in $100 \mathrm{mM}$ potassium phosphate, $\mathrm{pH} 7.4$ at $25^{\circ} \mathrm{C}$ for $2 \mathrm{~h}$; $\mathbf{B}$ ) absorbance spectra of $10 \mu \mathrm{M}$ P330L AADC before (dashed line) and after the addition of $2 \mathrm{mM}$ DME (colored lines) in $100 \mathrm{mM}$ potassium phosphate, $\mathrm{pH} 7.4$ at $25^{\circ} \mathrm{C}$ for $2 \mathrm{~h}$; C) HPLC analyses of coenzyme content following addition of $2 \mathrm{mM} \mathrm{DME}$ to $10 \mu \mathrm{M} \mathrm{WT}$ and P330L. Black and light black circles refer to PLP and PLP-DME adduct for the reaction of WT with DME; blue and light blue squares refer to PLP and PLP-DME adduct for the reaction of P330L with DME; brown and green squares represent the total amount of DME in the reaction with WT and P330L, respectively. Lines are drawn only to guide the eye. 


\section{References}

1. Sandmeier E, Hale TI, Christen P (1994) Multiple evolutionary origin of pyridoxal-5'phosphate-dependent amino acid decarboxylases. Eur J Biochem 221: 997-1002. DOI 10.1111/j.1432-1033.1994.tb18816.x

2. Paiardini A, Giardina G, Rossignoli G, Voltattorni CB, Bertoldi M (2017) New Insights Emerging from Recent Investigations on Human Group II Pyridoxal 5'-Phosphate Decarboxylases. Curr Med Chem 24: 226-244. DOI 10.2174/0929867324666161123093339 CMC-EPUB-79890 [pii]

3. Grishin NV, Phillips MA, Goldsmith EJ (1995) Modeling of the spatial structure of eukaryotic ornithine decarboxylases. Protein Sci 4: 1291-1304. DOI 10.1002/pro.5560040705

4. Jansonius JN (1998) Structure, evolution and action of vitamin B6-dependent enzymes. Curr Opin Struct Biol 8: 759-769. DOI 10.1016/s0959-440x(98)80096-1

5. Bertoldi M (2014) Mammalian Dopa decarboxylase: structure, catalytic activity and inhibition. Arch Biochem Biophys 546: 1-7. DOI 10.1016/j.abb.2013.12.020

6. Burkhard P, Dominici P, Borri-Voltattorni C, Jansonius JN, Malashkevich VN (2001) Structural insight into Parkinson's disease treatment from drug-inhibited DOPA decarboxylase. Nat Struct Biol 8: 963-967. DOI 10.1038/nsb1101-963

7. Giardina G, Montioli R, Gianni S, Cellini B, Paiardini A, Voltattorni CB, Cutruzzolà F (2011) Open conformation of human DOPA decarboxylase reveals the mechanism of PLP addition to Group II decarboxylases. Proc Natl Acad Sci U S A 108: 20514-20519. DOI 10.1073/pnas.1111456108

8. Torrens-Spence MP, Chiang YC, Smith T, Vicent MA, Wang Y, Weng JK (2020) Structural basis for divergent and convergent evolution of catalytic machineries in plant aromatic amino acid decarboxylase proteins. Proc Natl Acad Sci U S A 117: 10806-10817. DOI 10.1073/pnas.1920097117 9. Bertoldi M, Frigeri P, Paci M, Voltattorni CB (1999) Reaction specificity of native and nicked 3,4-dihydroxyphenylalanine decarboxylase. J Biol Chem 274: 5514-5521. DOI 10.1074/jbc.274.9.5514

10. Kass I, Hoke DE, Costa MG, Reboul CF, Porebski BT, Cowieson NP, Leh H, Pennacchietti E, McCoey J, Kleifeld O, et al. (2014) Cofactor-dependent conformational heterogeneity of GAD65 and its role in autoimmunity and neurotransmitter homeostasis. Proc Natl Acad Sci U S A 111: E2524-2529. DOI 10.1073/pnas.1403182111

11. Komori H, Nitta Y, Ueno H, Higuchi Y (2012) Structural study reveals that Ser-354 determines substrate specificity on human histidine decarboxylase. J Biol Chem 287: 29175-29183. DOI 10.1074/jbc.M112.381897

12. Bertoldi M, Gonsalvi M, Contestabile R, Voltattorni CB (2002) Mutation of tyrosine 332 to phenylalanine converts dopa decarboxylase into a decarboxylation-dependent oxidative deaminase. J Biol Chem 277: 36357-36362. DOI 10.1074/jbc.M204867200

M204867200 [pii]

13. Fenalti G, Law RH, Buckle AM, Langendorf C, Tuck K, Rosado CJ, Faux NG, Mahmood K, Hampe CS, Banga JP, et al. (2007) GABA production by glutamic acid decarboxylase is regulated by a dynamic catalytic loop. Nat Struct Mol Biol 14: 280-286. DOI 10.1038/nsmb1228

14. Montioli R, Paiardini A, Kurian MA, Dindo M, Rossignoli G, Heales SJR, Pope S, Voltattorni CB, Bertoldi M (2016) The novel R347g pathogenic mutation of aromatic amino acid decarboxylase provides additional molecular insights into enzyme catalysis and deficiency. Biochim Biophys Acta 1864: 676-682. DOI 10.1016/j.bbapap.2016.03.011

15. Tunyasuvunakool K, Adler J, Wu Z, Green T, Zielinski M, Žídek A, Bridgland A, Cowie A, Meyer C, Laydon A, et al. (2021) Highly accurate protein structure prediction for the human proteome. Nature 596: 590-596. DOI 10.1038/s41586-021-03828-1 
16. Jumper J, Evans R, Pritzel A, Green T, Figurnov M, Ronneberger O, Tunyasuvunakool K, Bates R, Žídek A, Potapenko A, et al. (2021) Highly accurate protein structure prediction with AlphaFold. Nature 596: 583-589. DOI 10.1038/s41586-021-03819-2

17. Ruff KM, Pappu RV (2021) AlphaFold and Implications for Intrinsically Disordered Proteins. J Mol Biol: 167208. DOI 10.1016/j.jmb.2021.167208

18. Brun L, Ngu LH, Keng WT, Ch'ng GS, Choy YS, Hwu WL, Lee WT, Willemsen MA, Verbeek MM, Wassenberg T, et al. (2010) Clinical and biochemical features of aromatic L-amino acid decarboxylase deficiency. Neurology 75: 64-71. DOI 10.1212/WNL.0b013e3181e620ae

19. Wassenberg T, Molero-Luis M, Jeltsch K, Hoffmann GF, Assmann B, Blau N, Garcia-Cazorla A, Artuch R, Pons R, Pearson TS, et al. (2017) Consensus guideline for the diagnosis and treatment of aromatic 1-amino acid decarboxylase (AADC) deficiency. Orphanet J Rare Dis 12: 12. DOI 10.1186/s13023-016-0522-z

20. Himmelreich N, Montioli R, Bertoldi M, Carducci C, Leuzzi V, Gemperle C, Berner T, Hyland K, Thöny B, Hoffmann GF, et al. (2019) Aromatic amino acid decarboxylase deficiency: Molecular and metabolic basis and therapeutic outlook. Mol Genet Metab 127: 12-22. DOI 10.1016/j.ymgme.2019.03.009

21. Montioli R, Dindo M, Giorgetti A, Piccoli S, Cellini B, Voltattorni CB (2014) A comprehensive picture of the mutations associated with aromatic amino acid decarboxylase deficiency: from molecular mechanisms to therapy implications. Hum Mol Genet 23: 5429-5440. DOI $10.1093 / \mathrm{hmg} / \mathrm{ddu} 266$

22. Montioli R, Cellini B, Borri Voltattorni C (2011) Molecular insights into the pathogenicity of variants associated with the aromatic amino acid decarboxylase deficiency. J Inherit Metab Dis 34: 1213-1224. DOI 10.1007/s10545-011-9340-6

23. Montioli R, Bisello G, Dindo M, Rossignoli G, Voltattorni CB, Bertoldi M (2020) New variants of AADC deficiency expand the knowledge of enzymatic phenotypes. Arch Biochem Biophys 682: 108263. DOI 10.1016/j.abb.2020.108263

24. Montioli R, Janson G, Paiardini A, Bertoldi M, Borri Voltattorni C (2018) Heterozygosis in aromatic amino acid decarboxylase deficiency: Evidence for a positive interallelic complementation between R347Q and R358H mutations. IUBMB Life 70: 215-223. DOI 10.1002/iub.1718

25. Montioli R, Battini R, Paiardini A, Tolve M, Bertoldi M, Carducci C, Leuzzi V, Borri Voltattorni C (2019) A novel compound heterozygous genotype associated with aromatic amino acid decarboxylase deficiency: Clinical aspects and biochemical studies. Mol Genet Metab 127: 132-137. DOI 10.1016/j.ymgme.2019.05.004

26. Longo C, Montioli R, Bisello G, Palazzi L, Mastrangelo M, Brennenstuhl H, de Laureto PP, Opladen T, Leuzzi V, Bertoldi M (2021) Compound heterozygosis in AADC deficiency: A complex phenotype dissected through comparison among heterodimeric and homodimeric AADC proteins. Mol Genet Metab. DOI 10.1016/j.ymgme.2021.08.011

27. Richards S, Aziz N, Bale S, Bick D, Das S, Gastier-Foster J, Grody WW, Hegde M, Lyon E, Spector E, et al. (2015) Standards and guidelines for the interpretation of sequence variants: a joint consensus recommendation of the American College of Medical Genetics and Genomics and the Association for Molecular Pathology. Genet Med 17: 405-424. DOI 10.1038/gim.2015.30

28. Verbeek MM, Geurtz PB, Willemsen MA, Wevers RA (2007) Aromatic L-amino acid decarboxylase enzyme activity in deficient patients and heterozygotes. Mol Genet Metab 90: 363369. DOI 10.1016/j.ymgme.2006.12.001

29. van Rumund A, Pavelka L, Esselink RAJ, Geurtz BPM, Wevers RA, Mollenhauer B, Krüger R, Bloem BR, Verbeek MM (2021) Peripheral decarboxylase inhibitors paradoxically induce aromatic L-amino acid decarboxylase. NPJ Parkinsons Dis 7: 29. DOI 10.1038/s41531-021-00172-z 30. Peterson E, Sober H (1954) Preparation of Crystalline Phosphorylated Derivatives of Vitamin B6. Journal of American Chemical Society 76: 169-175

31. Bertoldi M, Moore PS, Maras B, Dominici P, Voltattorni CB (1996) Mechanism-based inactivation of dopa decarboxylase by serotonin. J Biol Chem 271: 23954-23959 
32. Bertoldi M, Dominici P, Moore PS, Maras B, Voltattorni CB (1998) Reaction of dopa decarboxylase with alpha-methyldopa leads to an oxidative deamination producing 3,4dihydroxyphenylacetone, an active site directed affinity label. Biochemistry 37: 6552-6561. DOI 10.1021/bi9718898

bi9718898 [pii]

33. Sastry GM, Adzhigirey M, Day T, Annabhimoju R, Sherman W (2013) Protein and ligand preparation: parameters, protocols, and influence on virtual screening enrichments. J Comput Aided Mol Des 27: 221-234. DOI 10.1007/s10822-013-9644-8

34. Parra RG, Schafer NP, Radusky LG, Tsai MY, Guzovsky AB, Wolynes PG, Ferreiro DU (2016) Protein Frustratometer 2: a tool to localize energetic frustration in protein molecules, now with electrostatics. Nucleic Acids Res 44: W356-360. DOI 10.1093/nar/gkw304

35. Ferreiro DU, Komives EA, Wolynes PG (2014) Frustration in biomolecules. Q Rev Biophys 47: 285-363. DOI 10.1017/S0033583514000092

36. Jenik M, Parra RG, Radusky LG, Turjanski A, Wolynes PG, Ferreiro DU (2012) Protein frustratometer: a tool to localize energetic frustration in protein molecules. Nucleic Acids Res 40: W348-351. DOI 10.1093/nar/gks447

37. Rossignoli G, Grottesi A, Bisello G, Montioli R, Borri Voltattorni C, Paiardini A, Bertoldi M (2018) Cysteine 180 Is a Redox Sensor Modulating the Activity of Human Pyridoxal 5'-Phosphate Histidine Decarboxylase. Biochemistry 57: 6336-6348. DOI 10.1021/acs.biochem.8b00625

38. Bertoldi M, Voltattorni CB (2009) Multiple roles of the active site lysine of Dopa decarboxylase. Arch Biochem Biophys 488: 130-139. DOI 10.1016/j.abb.2009.06.019

39. Schnackerz KD, Tai CH, Simmons JW, Jacobson TM, Rao GS, Cook PF (1995) Identification and spectral characterization of the external aldimine of the $\mathrm{O}$-acetylserine sulfhydrylase reaction. Biochemistry 34: 12152-12160. DOI 10.1021/bi00038a008

40. Voltattorni CB, Minelli A, Dominici P (1983) Interaction of aromatic amino acids in D and L forms with 3,4-dihydroxyphenylalanine decarboxylase from pig kidney. Biochemistry 22: 22492254. DOI 10.1021/bi00278a030

41. Montioli R, Cellini B, Dindo M, Oppici E, Voltattorni CB (2013) Interaction of human Dopa decarboxylase with L-Dopa: spectroscopic and kinetic studies as a function of $\mathrm{pH}$. Biomed Res Int 2013: 161456. DOI 10.1155/2013/161456

42. Bertoldi M, Borri Voltattorni C (2003) Reaction and substrate specificity of recombinant pig kidney Dopa decarboxylase under aerobic and anaerobic conditions. Biochim Biophys Acta 1647: 42-47. DOI S1570963903000463 [pii]

43. Bertoldi M, Voltattorni CB (2001) Dopa decarboxylase exhibits low pH half-transaminase and high $\mathrm{pH}$ oxidative deaminase activities toward serotonin (5-hydroxytryptamine). Protein Sci 10: 1178-1186. DOI 10.1002/pro.101922

44. Bertoldi M, Cellini B, Maras B, Voltattorni CB (2005) A quinonoid is an intermediate of oxidative deamination reaction catalyzed by Dopa decarboxylase. FEBS Lett 579: 5175-5180. DOI S0014-5793(05)01014-8 [pii]

10.1016/j.febslet.2005.08.029

45. Bertoldi M, Cellini B, Montioli R, Borri Voltattorni C (2008) Insights into the mechanism of oxidative deamination catalyzed by DOPA decarboxylase. Biochemistry 47: 7187-7195. DOI $10.1021 / \mathrm{bi} 800478 \mathrm{~s}$

46. Moore PS, Bertoldi M, Dominici P, Borri Voltattorni C (1997) Aromatic amino acid methyl ester analogs form quinonoidal species with Dopa decarboxylase. FEBS Lett 412: 245-248. DOI 10.1016/s0014-5793(97)00788-6

47. Rossignoli G, Krämer K, Lugarà E, Alrashidi H, Pope S, De La Fuente Barrigon C, Barwick $\mathrm{K}$, Bisello G, Ng J, Counsell J, et al. (2021) Aromatic 1-amino acid decarboxylase deficiency: a patient-derived neuronal model for precision therapies. Brain. DOI 10.1093/brain/awab123 


\section{Statements and Declarations}

Acknowledgments Centro Piattaforme Tecnologiche of the University of Verona is acknowledged. The authors thank gratefully Silvia Bianconi for her skillful technical assistance.

Funding This work was supported by a grant from PTC Therapeutics and by FUR University of Verona to MB.

\section{Competing Interests}

The authors have no relevant financial or non-financial interests to disclose.

\section{Ethics approval}

The present study has been approved by local ethic committee (Ethics Committee of the University of Warsaw, Poland) and patient parents signed the informed consent for genetic analysis.

Author contributions GB performed the in vitro biochemical analyses. JP and GB carried out the bioinformatic analysis. KS and JSC clinically managed the patient and KK assessed the levels of catabolites in CSF and urine. MMV, MAAPW and RAW determined the plasma level of AADC. KWT and AMR performed the genetic analyses. GB, JP, AMR and JD contributed to data analyses. MMV, RAW and MB initiated this collaborative study. MB coordinated and supervised the work, analyzed and interpreted data and wrote the first draft of the manuscript. All authors contributed to the final version of the manuscript. All authors have approved the final version of the manuscript.

\section{Data Availability}

Data and material are available upon request. 


\section{Fig 1}

A)
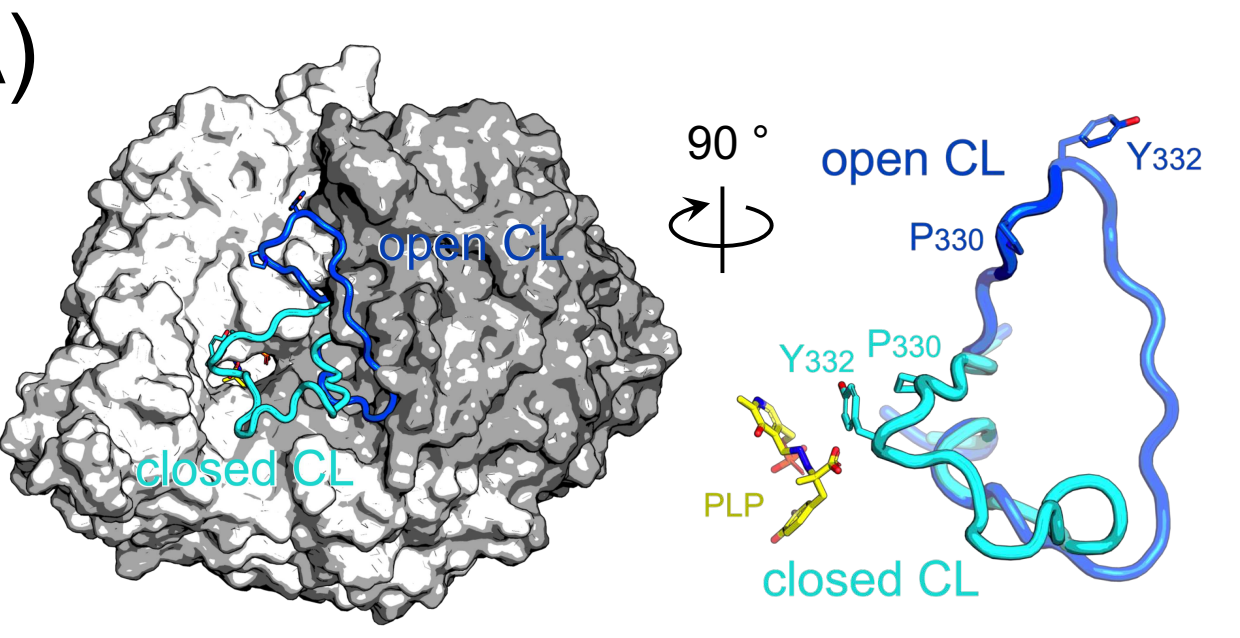

B)

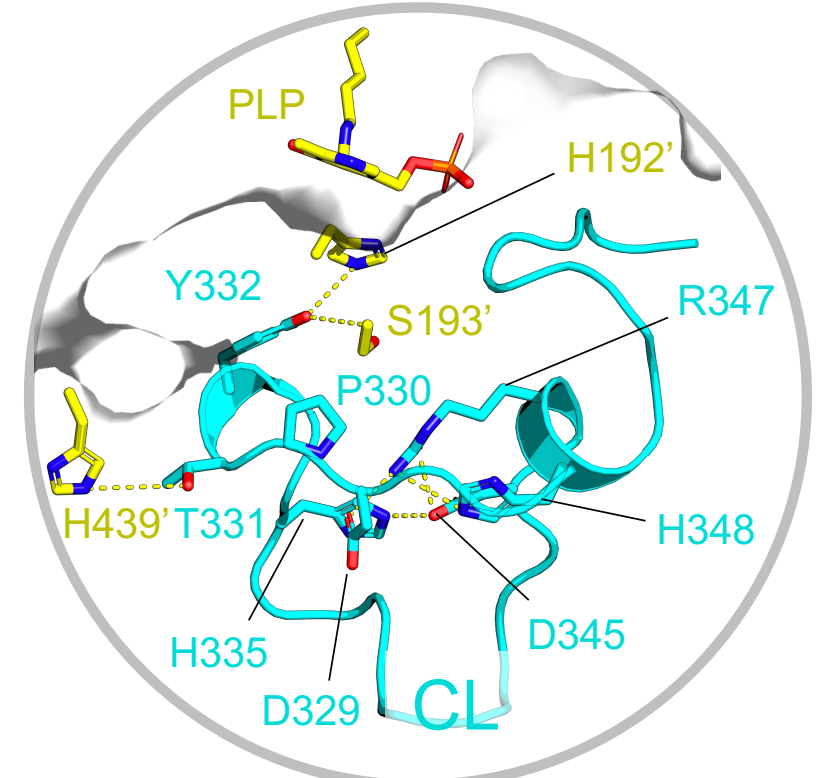

C)
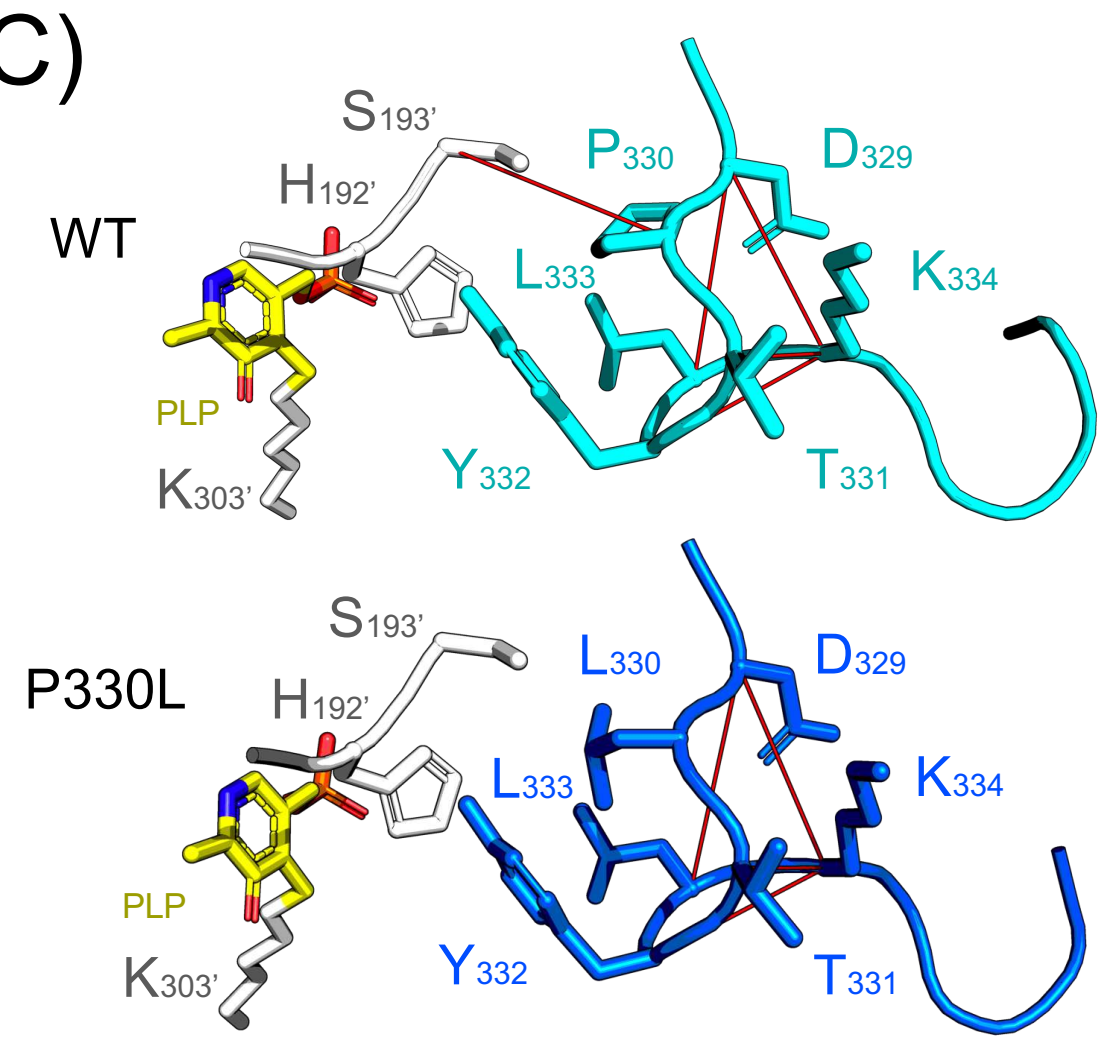

D)

WT

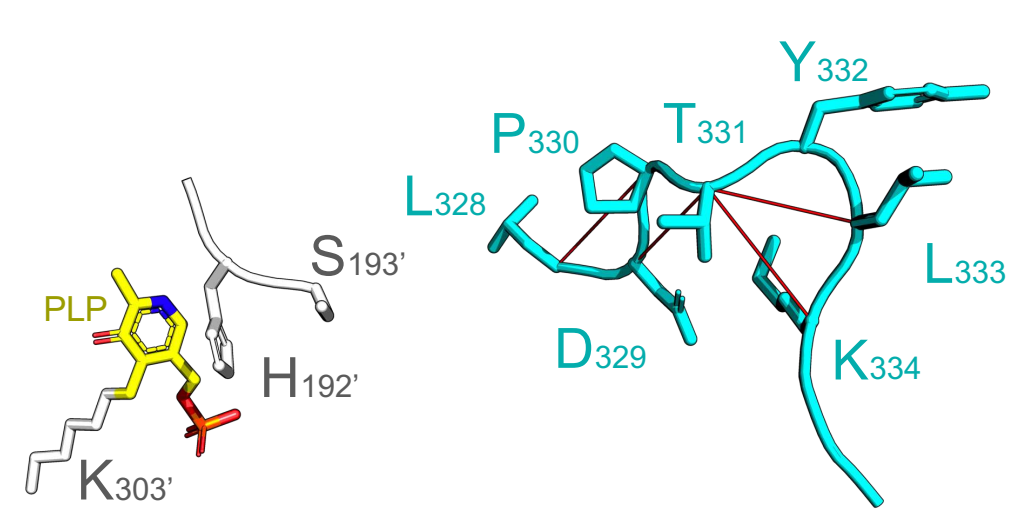

P330L

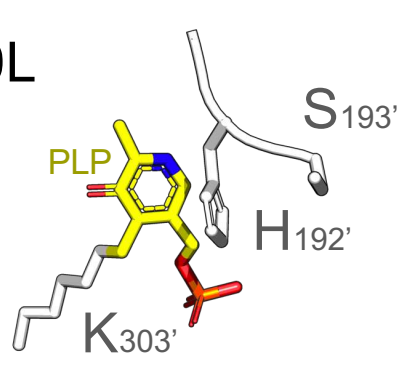

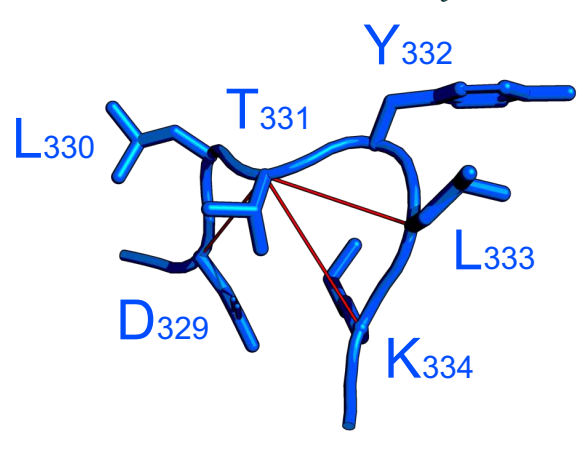


Fig 2

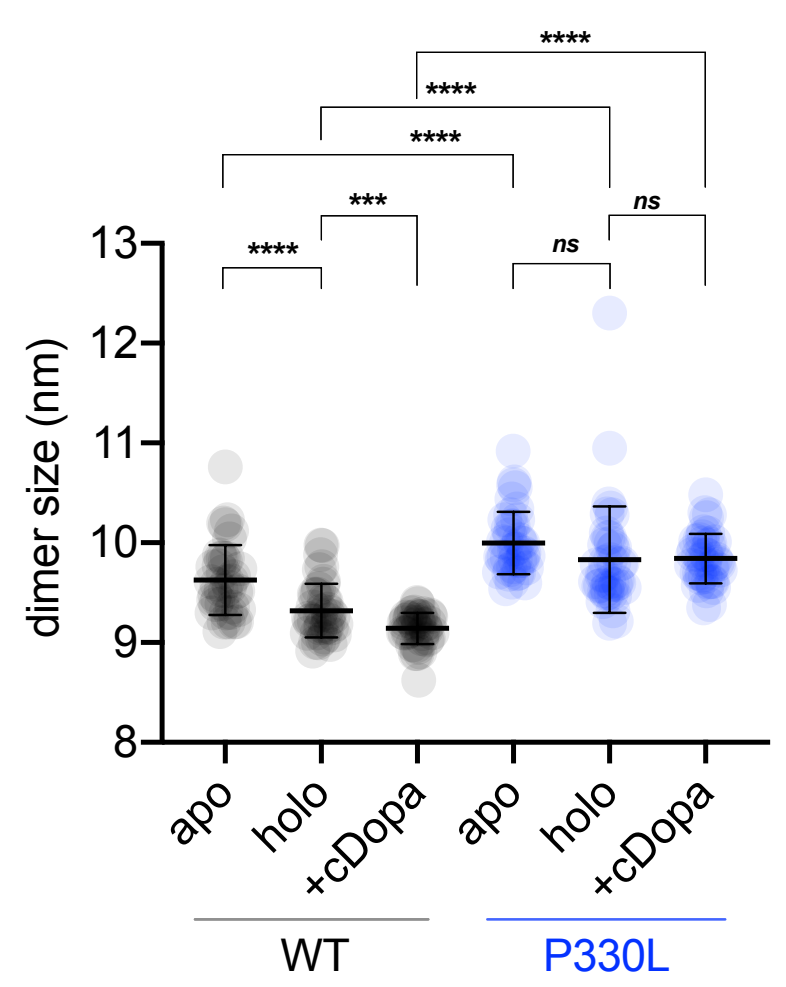


Fig 3

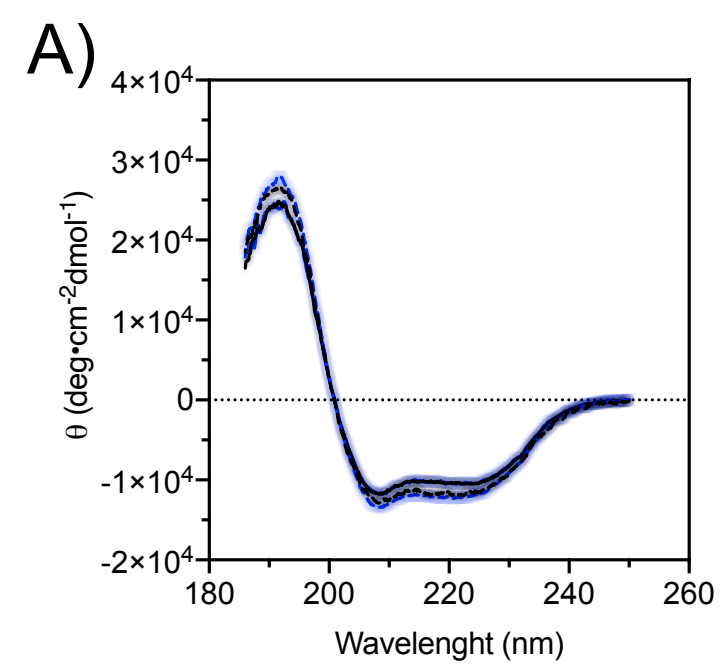

B)
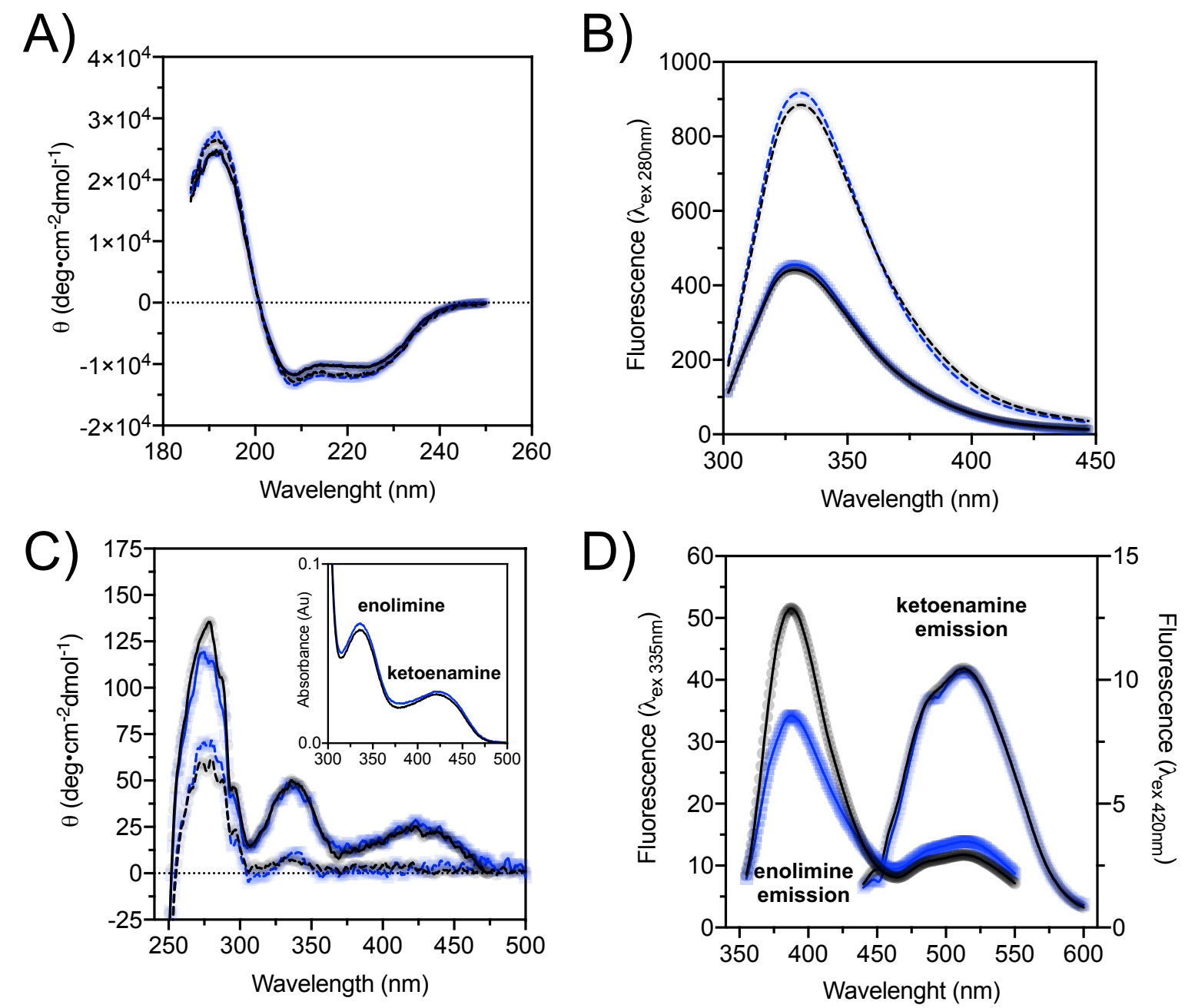


\section{Fig 4}

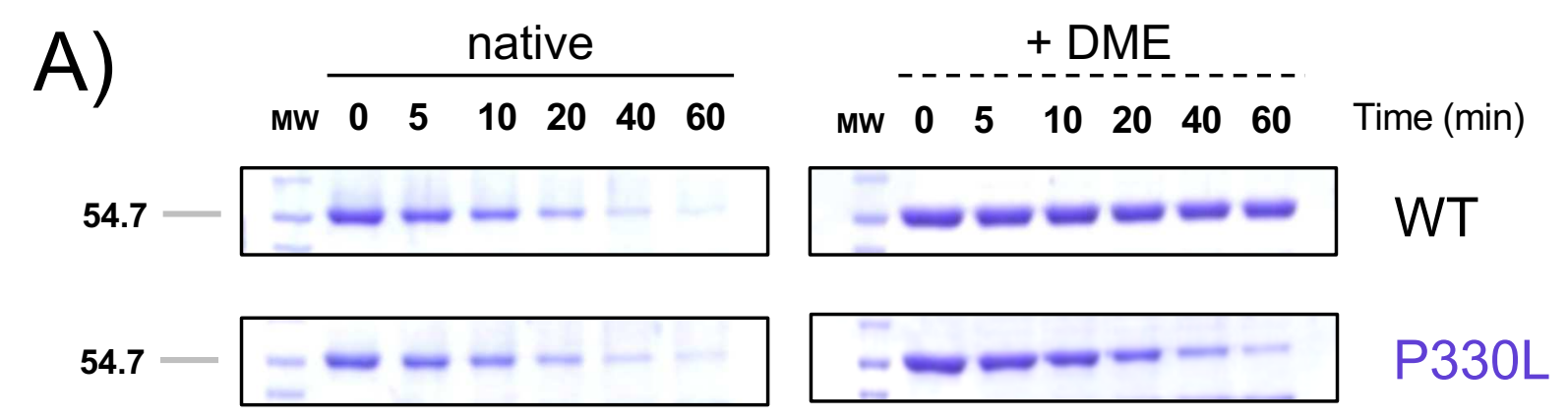

B)

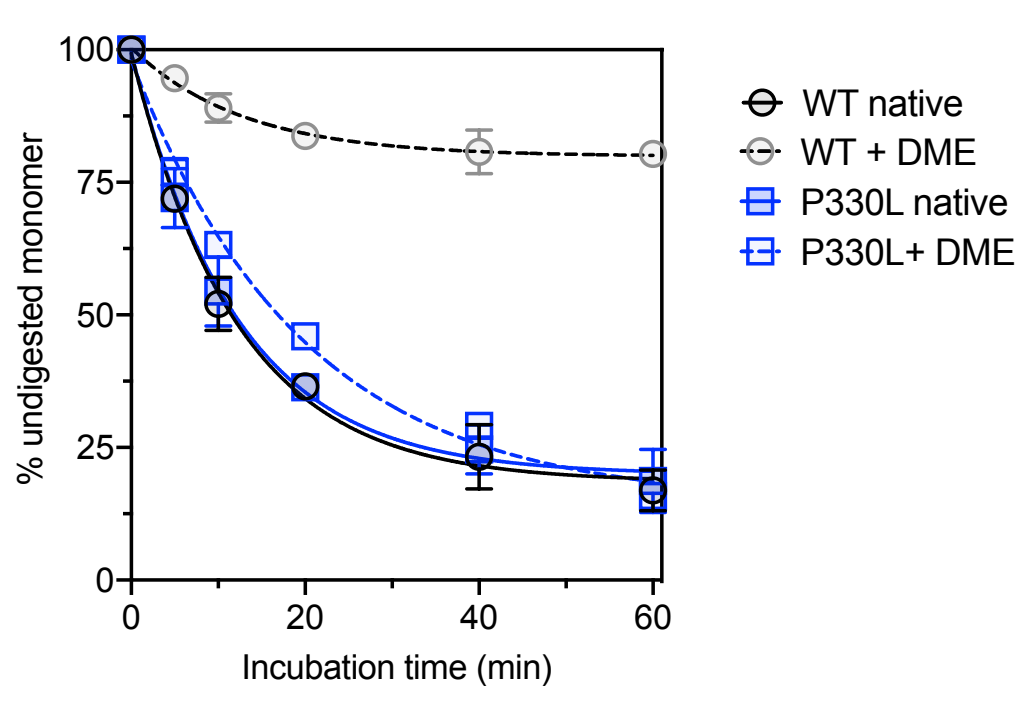


Fig 5
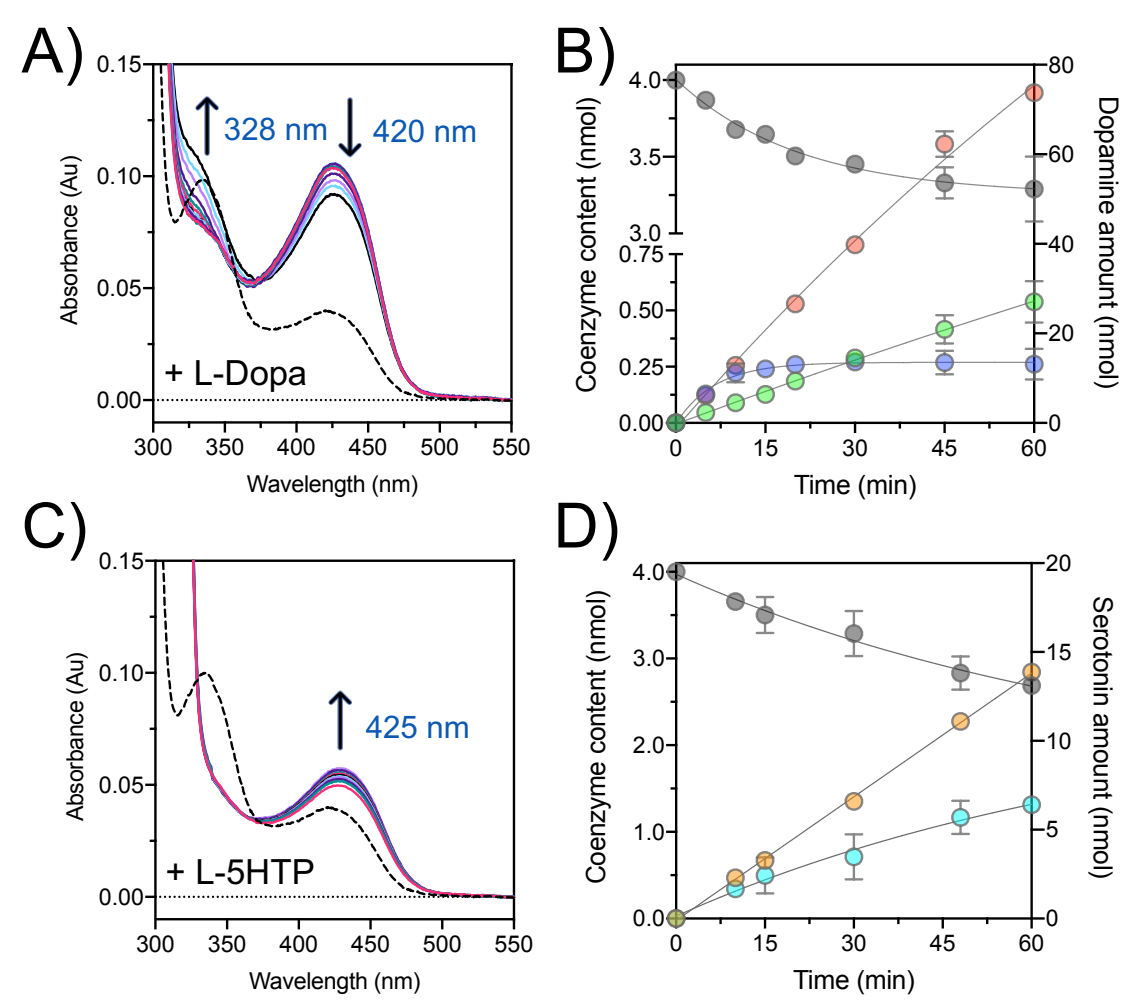

D)

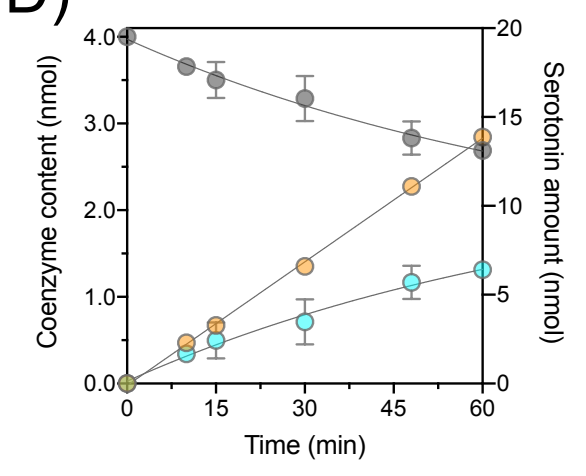




\section{FIG 6}
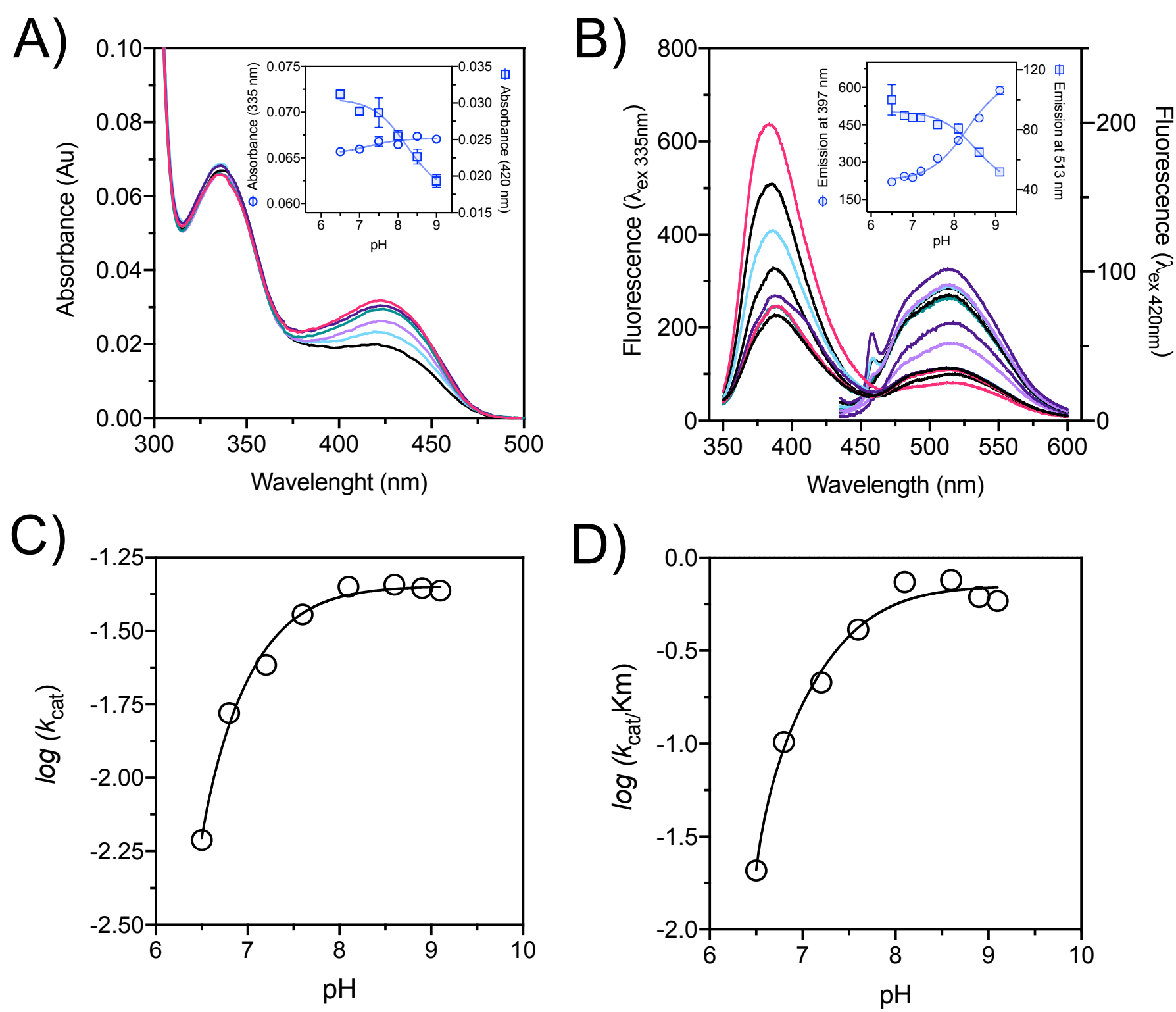


\section{FIG 7}
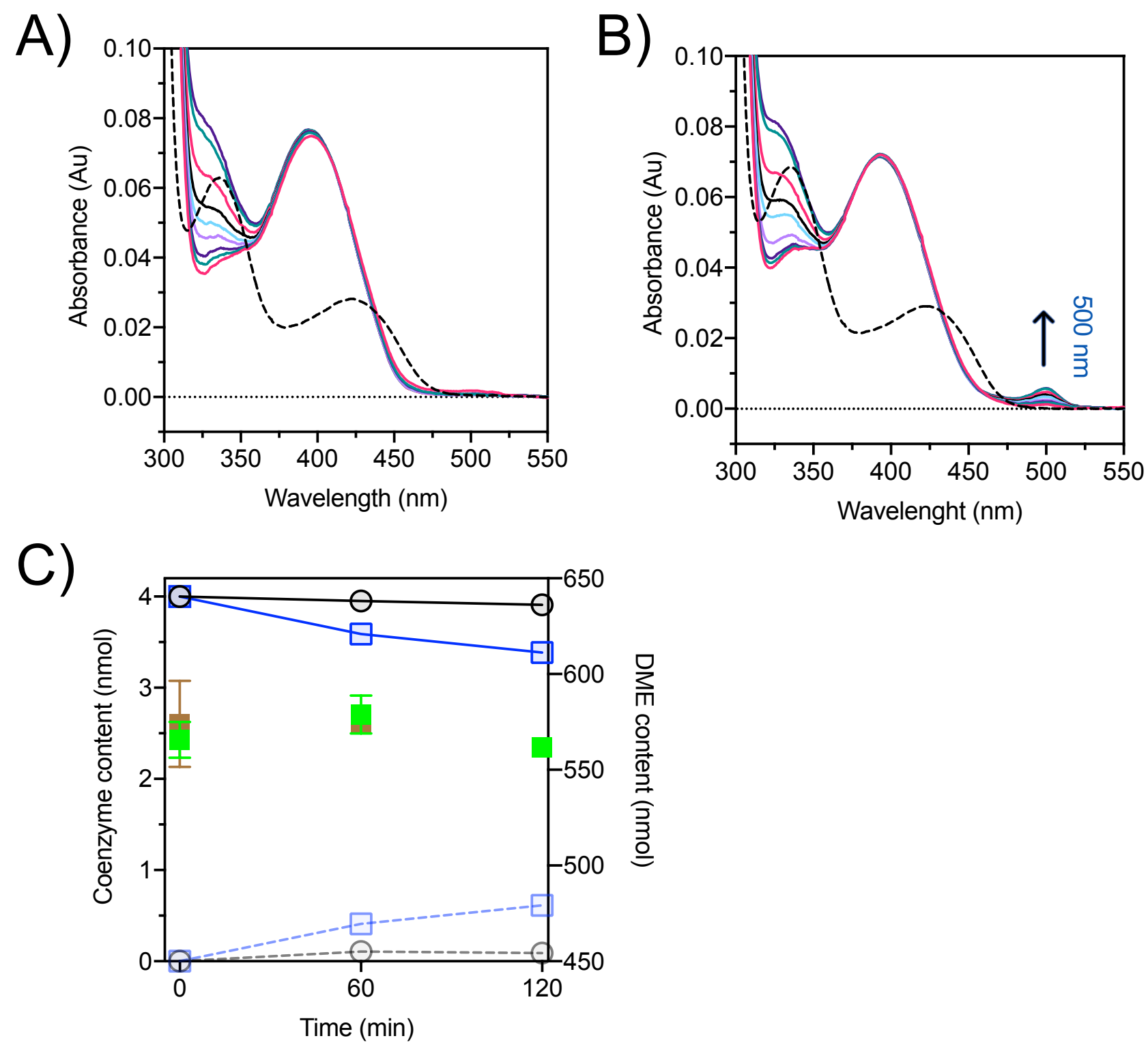


\section{Supplementary Files}

This is a list of supplementary files associated with this preprint. Click to download.

- SupplementalResults.pdf

- supplementalP330L.pdf 